\title{
Bio-inspired artemether-loaded human serum albumin nanoparticles for effective control of malaria-infected erythrocytes
}

\begin{abstract}
Aim: The intra-erythrocytic development of the malarial parasite is dependent on active uptake of nutrients, including human serum albumin (HSA), into parasitized red blood cells ( $p R B C s$ ). We have designed HSA-based nanoparticles as a potential drug-delivery option for antimalarials. Methods: Artemether-loaded nanoparticles (AANs) were designed and antimalarial activity evaluated in vitro/in vivo using Plasmodium falciparum/Plasmodium berghei species, respectively. Results: Selective internalization of AAN into Plasmodium-infected RBCs in preference to healthy erythrocytes was observed using confocal imaging. In vitro studies showed $50 \%$ dose reduction for AAN as compared with drug-only controls to achieve IC $\mathrm{C}_{50}$ levels of inhibition. The nanoparticles exhibited twofold higher peak drug concentrations in RBCs with antimalarial activity at $50 \%$ of therapeutic doses in P. bergei infected mice. Conclusion: Novel HSA-based nanoparticles offer safe and effective approach for selective targeting of antimalarial drugs.
\end{abstract}

First draft submitted: 10 June 2016; Accepted for publication: 30 August 2016; Published online: 19 October 2016

Keywords: artemether $\bullet$ human serum albumin nanoparticles $\bullet$ malaria $\bullet$ parasitized RBCs

Malaria is a global health priority with more than 3 billion people at risk [1]. Effective treatment of malaria is hampered by the development and rapid spread of resistance to all known classes of antimalarials. Furthermore, the nonspecific targeting of intracellular parasites result in high doses of therapeutic agents being used with higher incidence of related toxicities. Upon invasion by the malaria parasite, the host erythrocyte cell membrane undergoes major remodeling in structure, composition and function [2]. The intra-erythrocytic parasites import nutrients for their development and export proteins and lipids into the host cell cytoplasm. This transportation occurs through new permeability pathways, which selectively increases the permeability of the cell membrane of parasitized red blood cells ( $\mathrm{pRBCs}$ ) to a wide range of low molecular weight solutes [3]. An interconnected network of tubovesicular membranes is postulated to be responsible for the import of small molecules, whereas secretary cleft and lipid rafts may play a significant role in macromolecular transport in pRBCs [4]. Certain macromolecules such as dextran, Protein $A$ and IgG2 antibody are known to have direct access to the parasite through the 'parasitophorous duct', a tubular membranous structure that extends between the parasitophorous vacuolar membrane and the erythrocytic membrane [5-7]. These changes are of interest for antimalarial chemotherapy, as possible routes for targeting parasiticidal drugs into pRBCs.

Nanocarrier-based systems are providing new and powerful opportunities for targeting malaria-infected erythrocytes for the treatment of the disease $[8,9]$. The overall goal for effective antimalarial therapy is the achievement of high intracellular drug concentrations in pRBCs. This would require the
Aditi A Sidhaye', Kanchan C Bhuran', Sneha Zambare', Munna Abubaker ${ }^{2}$, Niroshini Nirmalan ${ }^{2}$ \& Kamalinder K Singh ${ }^{* 1,3}$

${ }^{1} \mathrm{CU}$ Shah College of Pharmacy, SNDT Women's University, Santacruz (W), Mumbai 400049, India

${ }^{2}$ School of Environment \& Life Sciences, University of Salford, Manchester, M5 4WT, UK

3School of Pharmacy \& Biomedical Sciences, University of Central Lancashire, Preston, PR1 2HE, UK *Author for correspondence: ksingh1@uclan.ac.uk

kksingh35@hotmail.com
Future
Medicine 
drug to cross multiple membrane barriers to access the intraparasitic targets, including the host erythrocyte membrane, the parasitophorous vacuolar membrane, the parasite plasma membrane and the food vacuolar membrane or endoplasmic reticulum membrane [10].

Human serum albumin (HSA) is a nontoxic, nonimmunogenic, biocompatible and biodegradable protein and has been widely used to design nanoparticles for drug delivery, and includes the commercially available nanoparticle albumin-bound (nab) paclitaxel, Abraxane $^{\mathrm{TM}}$ [11]. In addition, HSA, an endogenous transport protein with multiple drug binding sites can act as a versatile drug carrier and offer various possibilities of surface modification by appending ligands to modify pharmacokinetics, enhance stability, prolong circulation half-life, alter drug release or act as a targeting agent $[12,13]$. Notably high specificity and affinity interaction of native serum albumin with the parasitized erythrocytes have been reported [14]. Significantly, biotin-labeled and radio-iodinated HSA when added in vitro to malaria parasite cultures exhibited uptake, access to the parasite membrane through a membrane network and degradation in the parasite [15]. We took advantage of these features to use HSA as a nanocarrier for targeting antimalarial drugs to pRBCs. The use of HSA to prepare nanoparticles for antimalarial drugs is an adept concept for selective targeting to pRBCs.

Artemether (ATM), a fast acting blood schizonticide is used as the current first-line therapy for severe multidrug-resistant malaria [16]. However, a very short half-life and poor aqueous solubility limits the clinical efficacy of this drug [17]. Currently, an oil-based injectable formulation for intramuscular administration is available commercially. However, its use is limited due to its nondefined and erratic absorption. A suitable vehicle for intravenous delivery of ATM is urgently needed, especially for treating severe and cerebral malaria. HSA binds strongly to artemisinin and its derivatives through thiol and amino groups [18], paving the path to the development of ATM-loaded HSAbased nanoparticles, which will open up opportunity for intravenous administration of ATM.

The aim of the present research was to develop and characterize HSA-based ATM nanoparticles for parenteral delivery and investigate their antimalarial efficacy and selective targeting to pRBCs. These nanoparticles were fabricated systematically using Quality-by-Design $(\mathrm{QbD})$ approach. The nanoparticles were characterized for morphology, particle size, polydispersity index (PI), $\zeta$ potential, differential scanning calorimetry (DSC), x-ray diffraction (XRD) and Fourier transform infrared spectroscopy (FTIR) analysis. Their antimalarial efficacy was evaluated in vitro and in vivo using Plasmodium falciparum and Plasmodium berghei spe- cies, respectively. Pharmacokinetic studies after single intravenous injection were carried out in parasitized mice model to determine intracellular drug concentration in the RBCs. Confocal microscopy imaging was used to examine the entry of these nanoparticles selectively in $\mathrm{pRBC}$ in preference to noninfected RBCs. To best of our knowledge this is the first report of selective targeting of pRBCs using HSA-based nanoparticles.

\section{Experimental}

\section{Materials}

ATM was obtained as a gift sample from V. S. Internationals. HSA (Alburel) was purchased from Lifeline Pharmaceuticals, India. Absolute ethanol and polysorbate 80 were purchased from S. D. Fine chemicals Ltd., India. Rhodamine B and 4'6-diamino-2-phenylindole were purchased from Sigma Aldrich, India. Trehalose was gift sample from Signet Chemicals, India. SYBR Green, hypoxanthine, gentamycin, formaldehyde solution and albumin bovine serum fraction $\mathrm{V}$ were procured from Sigma Aldrich, UK. Dextrose anhydrous, was sourced from Fisher Scientific, UK and Hepes medium was from Gibco, Life Technologies, UK. All solvents and chemicals used were of analytical grade.

\section{In vitro culture of Plasmodium}

Plasmodiun falciparum parasites (strain 3D7 and K1) were grown in O+ve human RBCs in RPMI 1640 [+] L-glutamine, [+] Hepes medium supplemented with $5 \mathrm{mg} / \mathrm{l}$ albumin bovine serum fraction $\mathrm{V}, 50 \mathrm{mg} / \mathrm{l}$ hypoxanthine, $5 \mathrm{ml} / \mathrm{l}$ of $40 \%$ glucose and $50 \mathrm{mg} / \mathrm{l}$ of gentamycin (in phosphate buffered saline [PBS]) in accordance with Read and Hyde [19]. Continuous culture of parasites at $5 \%$ hematocrit was carried out in 25 or $12.5 \mathrm{~cm}^{2}$ flasks, in final culture volumes of 10 and $5 \mathrm{ml}$, respectively, with subculturing at 48 and $72 \mathrm{~h}$ intervals. Synchronization of parasite stages was achieved by adding sorbitol solution $(5 \% \mathrm{w} / \mathrm{v}$ in distilled water filtered through a $0.22 \mu \mathrm{m}$ filter) to the parasite pellet with a $5 \mathrm{~min}$ incubation. The culture was centrifuged at $3000 \mathrm{rpm}$ for $5 \mathrm{~min}$ and the supernatant discarded. The pellet was washed three-times in complete RPMI prior to resuspension at the appropriate hematocrit. Preliminary estimation of parasitemia was carried out using Giemsa-stained thin blood smears. Culture flasks were gassed with a $5 \% \mathrm{CO}_{2}$, $5 \% \mathrm{O}_{2}, 90 \% \mathrm{~N}_{2}$ air mixture (BOC Limited, UK) and incubated at $37^{\circ} \mathrm{C}$ (Leec $190 \mathrm{CO}_{2}$, Leec Limited, UK).

\section{In vivo culture of Plasmodium}

The $P$. berghei ANKA strain was used for in vivo evaluation of antimalarial activity. The strain is well characterized with high mortality in mice. The parasites kept in liquid nitrogen were thawed (at $37^{\circ} \mathrm{C}$ ) 
and parasitic culture was maintained in live mice through serial blood transfusion in fresh animals every 4 days. Animal experiments were carried out as per the protocols (CUSCP/IAEC/43/2012 and CUSCP/ IAEC/31/2013) approved by Institutional Animal Ethics Committee of SNDT Women's University, Mumbai. Research was conducted in accordance with guidelines on animal use and care and followed internationally accepted principles for laboratory animal use and care. Swiss albino mice of either sex aged 9-10 weeks and weighing 22-27 g were used for the experiment. The animals were quarantined at a temperature of $22 \pm 3^{\circ} \mathrm{C}$ and $65 \%$ relative humidity and were fed a standard pelleted diet and provided with clean drinking water, throughout the experiment.

\section{Development of artemether-loaded HSA nanoparticles}

Artemether-loaded HSA nanoparticles (AANs) were prepared using the desolvation technique [20-22], followed by high-pressure homogenization. Briefly, HSA (Alburel) was diluted with water to obtain the desired concentration $(2 \% \mathrm{w} / \mathrm{v})$ and $\mathrm{pH}$ was maintained at neutral. The drug was dissolved in a mixture of ethanol $(40 \% \mathrm{v} / \mathrm{v})$ and polysorbate $80(3 \% \mathrm{w} / \mathrm{v})$, which was slowly injected into the protein solution. The dispersion was cross-linked by heating and homogenized in a high-pressure homogenizer (Niro Saovi Panda Plus, Italy). The nanoparticles thus obtained were lyophilized in presence of trehalose as cryoprotectant in a freeze dryer (Martin Christ Epsilon 2-4LSC, Germany). A 48-h cycle was divided into three phases of 6,18 and $24 \mathrm{~h}$ for the stages of freezing, main drying and final drying, respectively (Supplementary Table 1). Blank nanoparticles (BAN) were prepared using exactly the same protocol without addition of any drug. Rhodamine B $(2.5 \mu \mathrm{g})$ was covalently bound to AAN $(1 \mathrm{ml})$ using 1-ethyl-3-(3-dimethylaminopropyl) carbodiimide hydrochloride/N-hydroxysulfo-succinimide reaction with incubation at room temperature for $3 \mathrm{~h}$ to prepare rhodamine-labeled nanoparticles $[23,24]$. The labeled nanoparticles were subjected to dialysis against deionized water to remove any excess dye. Briefly $1 \mathrm{ml}$ Rhodamine $\mathrm{B}$ tagged nanoparticles were put in dialysis membrane (3K MWCO) bag and suspended in beaker filled with $1000 \mathrm{ml}$ deionized water with continuous magnetic stirring for $1 \mathrm{~h}$.

Systematic optimization of the formulation was carried out using the QbD approach [25]. The Ishikawa diagram was used to determine the critical quality attributes (CQAs) of the entire process (Figure 1). The method was optimized for materials, process and environment related factors (Table 1) to correct the CQAs during the development. Apart from conventional optimization with one factor at a time, two $3^{2}$ factorial designs (nine runs each) were applied to optimize the material related parameters. Design I investigated the effect of HSA (at three levels; 1,2 and $3 \% \mathrm{w} / \mathrm{v}$ ) and solvent concentration (at three levels; 20, 30 and $40 \% \mathrm{v} / \mathrm{v})$ and design II investigated the effect of HSA (at three levels; 1, 2 and 3\% w/v) and surfactant concentration (at three levels; 3, 5 and 7\% w/v) on the particle size of the nanoparticles (Table 2). Design Expert 8.0 was used to build response surface curves. Process related parameters, in other words, homogenization pressure and number of homogenization cycles were optimized by varying the homogenization pressure from 200 to 1400 bars with 1-20 homogenization cycles (20 runs; Supplementary Table 2). Environmentrelated factors such as $\mathrm{pH}$, type and concentration of cryoprotectant were also optimized.

\section{Fluorescence spectroscopy for determination of binding of ATM to HSA}

Fluorescence spectroscopy helps in understanding the residues that are involved in the drug-carrier interaction. Fluorescence spectra of HSA alone and in the presence of different concentrations of ATM were taken at excitation wavelength ( $\lambda$ ex $)$ of $280 \mathrm{~nm}$ and emission wavelength $(\lambda \mathrm{em})$ from 290 to $400 \mathrm{~nm}$ on Perkin Elmer LS55 fluorescence spectrometer. Fluorescence spectra of blank HSA nanoparticles (BAN) and AAN were also recorded. The fluorescence quenching data were analyzed according to the Stern-Volmer equation $[26,27]$ :

$\mathrm{F} 0 / \mathrm{F}=1+\mathrm{Ksv}[\mathrm{Q}]$

F0 and F denote fluorescence intensities in the absence and presence of quenchers, respectively. Ksv is the Stern-Volmer constant and [Q], the quencher concentration.

\section{Characterization of AANs}

\section{Particle size \& PI}

AAN was characterized for mean particle size and PI using photon correlation spectroscopy technique employing the N5 Beckman particle size analyzer (CA, USA). All measurements were carried out at a fixed angle of $90^{\circ}$ at $20^{\circ} \mathrm{C}$ using double distilled water as dispersant. The averaged values three measurements are presented.

\section{Morphology}

The microstructures of AAN particles were observed using transmission electron microscope (TEM; Philips CM 200,SAIF, IIT, Mumbai). Initially, sample diluted with double-distilled water was deposited on 


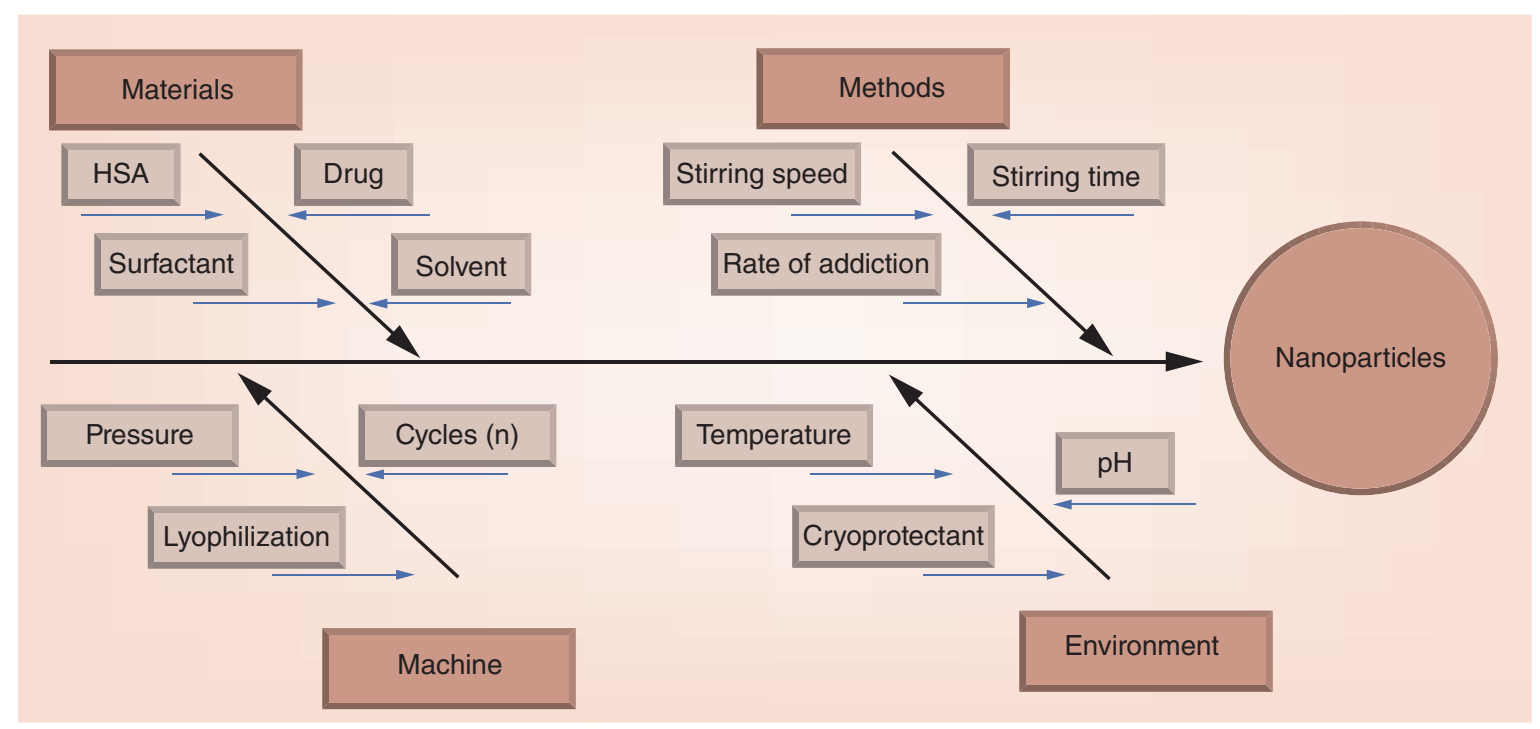

Figure 1. Ishikawa diagram for essential product and process parameters.

film-coated copper grids and allowed to dry overnight at room temperature. The dried samples were visualized under TEM. Size and morphology of lyophilized nanoparticles were examined using a field emission scanning electron microscope (SEM; Model JEOL JSM-7600F, MA, USA). AAN particles were first coated in gold to minimize the effect of heat during high power magnification.

\section{Differential scanning calorimetry}

DSC measurements on ATM, HSA and AAN were performed using a Q200 TA instrument (DE, USA). The heating runs were performed from 30 to $200^{\circ} \mathrm{C}$ at $10.0^{\circ} \mathrm{C} / \mathrm{min}$. An empty aluminum pan was used as a reference.
Fourier transform infrared spectroscopy

The interaction between the drug and carrier and the encapsulation of drug was evaluated using FTIR spectroscopy using JASCO FTIR 5300 Spectrometer with TGS detector (MD, USA) over the scanning wavelength range of $4000-400 \mathrm{~cm}^{-1}$. Potassium bromide disc method was employed to obtain FTIR spectrums of AAN as well as the individual components.

\section{X-ray diffraction studies}

XRD studies of ATM and AAN were performed using Philips Expertpro MPD diffractrometer (PAN analytical Inc., Germany) with resolution of $0.001 \AA$. The samples were radiated using a Cu target $(\lambda=1.54056 \AA)$ and scanning angles ranged from $7^{\circ}$ to $70^{\circ}$ of $2 \theta$.

\begin{tabular}{|c|c|c|c|c|c|}
\hline \multirow{2}{*}{\multicolumn{2}{|c|}{ Risk assessment related to }} & \multicolumn{4}{|c|}{ Critical quality attributes } \\
\hline & & Particle size & $\begin{array}{l}\text { Polydispersity } \\
\text { index }\end{array}$ & $\begin{array}{l}\text { Drug } \\
\text { entrapment }\end{array}$ & Stability \\
\hline \multirow[t]{4}{*}{ Materials } & HSA concentration & High & High & High & Medium \\
\hline & Drug:HSA ratio & Medium & Medium & High & Low \\
\hline & Solvent concentration & High & High & Low & Medium \\
\hline & Surfactant concentration & Medium & Medium & High & High \\
\hline \multirow[t]{2}{*}{ Process } & Homogenization pressure & High & High & Medium & High \\
\hline & Homogenization cycles & Medium & Medium & Medium & Medium \\
\hline \multirow[t]{2}{*}{ Environment } & $\mathrm{pH}$ & High & High & Low & Medium \\
\hline & Cryoprotectant & High & High & Low & Medium \\
\hline
\end{tabular}




\section{Determination of entrapment efficiency}

Entrapment efficiency was determined by separating the nanoparticles from aqueous dispersion by ultracentrifugation $\left(30,000 \times g\right.$ at $4^{\circ} \mathrm{C}$ for $\left.1 \mathrm{~h}\right)$ and the amount of free drug in the clear supernatant was analyzed by UV-spectroscopy (UV 2450, Shimadzu, Japan) [16]. The encapsulation efficiency was calculated by using the following equation:

$$
\frac{\mathrm{W}-\mathrm{W}}{\mathrm{W}} \times 100
$$

where $\mathrm{W}=$ amount of ATM initially added and $\mathrm{w}=$ amount of ATM present in the supernatant.

\section{In vitro drug release}

In vitro drug release study was performed in modified USP type II apparatus (Electrolab India, Pvt. Ltd, Mumbai, India). A total of $3 \mathrm{ml}$ of AAN dispersion or reconstituted lyophilized AANs were enclosed within cellulose acetate dialysis bag ( $3 \mathrm{~K} \mathrm{MWCO}$ ) and placed in $200 \mathrm{ml}$ of phosphate buffered saline ( $\mathrm{pH} \mathrm{7.4)}$ and ethanol (50:50) as dissolution medium to maintain sink conditions The medium was continuously stirred at $100 \mathrm{rpm}$ and maintained at $37 \pm 5^{\circ} \mathrm{C}$. A total of $5 \mathrm{ml}$ aliquots were withdrawn at predetermined time points $(0.5,1,2,4,6,8$ and $24 \mathrm{~h})$ and were replaced with the same volume of dissolution media. The concentration of drug released was analyzed by UV Spectroscopy (UV 2450, Shimadzu, Japan) [17].

\section{In vitro erythrocyte toxicity study}

Infection with $P$. falciparum results in erythrocyte rupture during its intra-erythrocytic life cycle causing hemolysis leading to anemia in severe conditions [28]. For an antimalarial drug delivery system, it is important to have action targeted specifically on pRBCs with no harm to the uninfected erythrocytes. The in vitro erythrocyte toxicity study determines the toxicity potential of the system on the uninfected erythrocytes on intravenous administration.

The test was performed using varying dilutions of AAN in normal saline $(1 \times, 10 \times$ and $100 \times)$. Polysorbate $80(3 \% \mathrm{w} / \mathrm{v})$ and Triton-X $100(2 \% \mathrm{w} / \mathrm{v})$ in normal saline were used as reference and positive control respectively. A total of $100 \mu \mathrm{l}$ of the prepared human RBCs were incubated with $900 \mu$ l of the test solutions for $30 \mathrm{~min}$. The reaction was stopped by addition of glutaraldehyde. The tubes were centrifuged to separate the debris and the supernatant was analyzed for the hemolytic activity by measuring the absorbance at 540 nm by UV spectrophotometry (UV 2450, Shimadzu, Japan). The percent hemolysis was calculated by the formula:

$\%$ Haemolysis =

$\frac{\text { Absorbance of sample - absorbance of control }}{\text { Absorbance of Triton X100 - absorbance of control }} \times 100$

In vitro susceptibility assay

Antimalarial drugs are maximally active on the mature trophozoite stage of parasite development. In vitro susceptibility has been correlated with the initial stage of the parasite, with isolates predominantly at the trophozoite stage having a twofold increase in $\mathrm{IC}_{50} \mathrm{~s}$ compared with those of parasites predominantly at the ring stage $[29,30]$. The in vitro antimalarial efficacy was therefore assessed using synchronized trophzoite stage cultures of $P$. falciparum (K1) parasite strains reported previously [31]. A total of $5 \mathrm{mM}$ stock solution of ATM (MW 298.38) in DMSO (ATM) was used to produce a dilution series for the dose response curve. AANs and BANs were reconstituted aseptically with sterile-filtered double-distilled water. A total of $0.2 \mathrm{ml}$ of the $P$. falciparum culture $(5 \%$ hematocrit and $0.5 \%$ parasitemia) was seeded in a 24-well plate and formulations and drug solution added along with complete media. The drug dose range was tested at 0.78, 1.56, $3.13,6.25,12.5,25$ and $50 \mathrm{nM}$ concentrations. The plates were incubated for $48 \mathrm{~h}$ at $37^{\circ} \mathrm{C}$ and parasitemia was determined by SYBR green-based flow cytometry methods as described previously [31]. Briefly, triplicate drug/nanoparticle-treated parasite cultures (100 $\mu \mathrm{l}$ each) are transferred to Eppendorf tubes and washed in $1 \mathrm{ml}$ PBS, and centrifuged at $1400 \mathrm{rpm}$ for $90 \mathrm{~s}$. The pellet is resuspended in $1 \mathrm{ml} 2.5 \times$ SYBR Green 1 and incubated in the dark for $20 \mathrm{~min}$ at room temperature. Samples were centrifuged as described and resuspended in $250 \mu \mathrm{l}$ of a fixative solution of $0.4 \%$

Table 2. Concentration levels of the dependent variables in factorial designs.

Factors

\begin{tabular}{|llll|} 
& -1 & 0 & +1 \\
\hline HSA $(\% \mathrm{w} / \mathrm{v})$ & 1 & 2 & 3 \\
\hline Solvent $(\% \mathrm{v} / \mathrm{v})$ & 20 & 30 & 40 \\
\hline Polysorbate $80(\% \mathrm{w} / \mathrm{v})$ & 3 & 5 & 7 \\
\hline HSA: Human serum albumin. & & & \\
\hline
\end{tabular}


formaldehyde solution and incubated at $4^{\circ} \mathrm{C}$ for 15 min. The pellets were washed three-times in PBS and suspended in $1 \mathrm{ml}$ of PBS for flow cytometric analysis on a BD FACSVerse flow cytometer (CA USA). A total of 50,000 events were recorded for each sample using the FITC channel and fluorescent intensity recorded. Percent inhibition was calculated against the drug-free control.

\section{Determination of cellular uptake of nanoparticles by confocal microscopy}

Synchronized ring stage parasite cultures $(200 \mu \mathrm{l})$ were incubated with Rhodamine B-labeled AAN $(25 \mu \mathrm{l})$ along with complete media $(1 \mathrm{ml})$ in a 24 -well cell culture plate, at $37^{\circ} \mathrm{C}$. The cellular uptake was observed at predetermined time points of $15 \mathrm{~min}, 1 \mathrm{~h}$ and $24 \mathrm{~h}$. The cells were washed with RPMI-1640 culture medium and stained with parasite nucleic acid stain 4'6-diamino-2-phenylindole (DAPI) for $20 \mathrm{~min}$ to distinguish pRBCs from normal RBCs. At each of the time points, a small sample $(5 \mu \mathrm{l})$ was removed from a stock culture of pRBC and wet mounted on a clean glass slide with a coverslip placed on it for examination. The cellular uptake was observed at a magnification of $100 \times$ using confocal laser scanning microscope (ZEISS LSM 510 META, Germany) at an excitation wavelength of $540 \mathrm{~nm}$ and an emission maximum of $625 \mathrm{~nm}$. As a positive control, pRBCs were stained with DAPI to distinguish them from uninfected RBCs and observed at excitation maximum of $358 \mathrm{~nm}$ and emission maximum of $461 \mathrm{~nm}$.

\section{In vivo antimalarial efficacy in P. berghei infected mice}

The lethal ANKA strain of $P$. berghei was used for the experiments [32]. Mice of either sex (average weight 25 g) were infected by intraperitoneal inoculation of the parasite obtained from the donor mouse blood diluted with citrate phosphate dextrose containing approximately $5 \times 10^{7}$-infected RBCs $/ \mathrm{ml}$. The mice were randomly divided into various groups $(n=5)$ (Table 4). Post infection, the animals were treated with AAN aseptically reconstituted using sterile saline at two dose levels $0.624 \mathrm{mg} /$ day and $0.312 \mathrm{mg} /$ day (equivalent to 100 and $50 \%$ of therapeutic dose of ATM) for four consecutive days. In lack of marketed intravenous ATM injection, artesunate (ARS), which has been previously employed for comparison in developmental antimalarial intravenous products [33,34], was used as reference. Larinate, marketed intravenous ARS injection, was reconstituted using the provided diluent under aseptic conditions and injected at same dose levels as AAN. BANs were administered in volume equivalent to AAN containing $0.624 \mathrm{mg}$ of drug in
AAN. On day 0, 1, 5, 7, 10, 14 and 21day thin blood smears were prepared by collecting a drop of blood from the tail vein. The blood smears were air-dried, fixed in methanol and stained with Giemsa stain. The smears were then washed, air-dried and observed under the microscope using an oil immersion lens and parasitemia was assessed. The study parameters involved number of surviving animals from each group on day 30 mean percent parasitemia over time (in days) for all groups. Data were expressed as mean $\pm S D$ and assessed by unpaired t-test using the Graph-pad Instat Demo version. A difference of $\mathrm{p}<0.05$ was considered as significant.

\section{Pharmacokinetics in P. berghei infected mice}

The parasitized animals were divided into three groups of six mice per group. When animals reached parasitemia of $50-60 \%$ the first group was administered AAN as single dose $(35 \mathrm{mg} / \mathrm{kg}$ equivalent amount of ATM) intravenous injection. ATM-free drug being insoluble in water its solution was prepared by dissolving it in nonclinical hydroaholic vehicle (ATM) [35] and administered intravenously to the second group while third group acted as control. At stipulated time intervals of $10 \mathrm{~min}, 30 \mathrm{~min}, 1,2,4,8,10$ and $24 \mathrm{~h}$, the animals were exposed to inhalation of anesthetic ether and blood samples were withdrawn using retroorbital puncture. The blood samples were centrifuged at $2000 \mathrm{rpm}$ for $10 \mathrm{~min}$ and the supernatant plasma and RBCs were collected in two separate clean Eppendorf tubes. To the $200 \mu \mathrm{l}$ plasma sample, $100 \mu \mathrm{l}$ of internal standard (artemisinin) and $700 \mu \mathrm{l}$ of acetonitrile were added and the contents of the tube were vortexed and then centrifuged at $2000 \mathrm{rpm}$ for 10 min using mini centrifuge (Hoefer, MA, USA) [36]. Similarly for extraction from RBCs, to $200 \mu$ of RBC sample, $100 \mu \mathrm{l}$ of internal standard, $20 \mu \mathrm{l}$ ammonium chloride buffer (for hemolysis of RBCs) and $680 \mu \mathrm{l}$ of acetonitrile were added. The mixture was vortexed and centrifuged at $2000 \mathrm{rpm}$ for $10 \mathrm{~min}$ (Hoefer mini centrifuge). The upper organic layer from each of the extractions was filtered and analyzed using HPLC (Agilent 1200 series, Singapore) equipped with C18 column (Princeton SPHER ODS, $250 \times 4.0 \mathrm{~mm} ; 5 \mu \mathrm{l}$ ) with PDA detector. Acetonitrile: water $(75: 25 \mathrm{v} / \mathrm{v})$ was used as mobile phase at flow rate of $0.7 \mathrm{ml} / \mathrm{min}$ and detection wavelength was set at $210 \mathrm{~nm}$ [37]. Each solution was injected in triplicate and mean peak areas were recorded for quantification for the drug.

\section{Results}

\section{Development of AANs}

Basic methodology and all the essential material and process parameters required for development of 
nanoparticles were derived in reference to the Ishikawa Diagram (Figure 1). Risk identification and risk analysis of material, process and environment variables (Table 1) provided parameters for control strategy for the CQAs [25]. Optimization of the formulation was performed to reduce the risk of the CQAs to obtain a robust and reproducible formulation.

Concentration of HSA and Drug: HSA ratio was optimized based on the particle size and entrapment efficiency of the drug. Increasing the HSA concentration resulted in increase in particle size (Figure 2A); hence, $2 \% \mathrm{w} / \mathrm{v}$ concentration was found to be optimum showing negligible drug precipitation and lower particle size [20]. Incorporating ATM at drug:HSA ratio of 1:5 resulted in precipitation of drug while 1:20 ratio led to increase in particle size without added advantage in loading efficiency thus drug:HSA ratio of 1:10 was found optimum (Table 3). ATM being highly water insoluble drug, incorporation of polysorbate 80 stabilized the nanoparticles with positive effect on drug entrapment (Figure 2C) [38,39]. Concentrations of HSA, ethanol and polysorbate 80 were further optimized using two $3^{2}$ full factorial designs. The p-values of 0.0009 and 0.0274 indicated that the models were significant. Reducing the HSA concentration and increase in ethanol concentration resulted in smaller particle size (Figure 2D) [40]. The blue region in the response surface curve (Figure 2E) shows the area of lowest particle size, which could be achieved at the optimum HSA and surfactant concentration levels.

Increasing the $\mathrm{pH}(>7)$ of the HSA solution prior to desolvation decreased the particle size of the nanoparticles (Figure 2B), due to increased repulsion between the molecules preventing their aggregation. This observation is in good agreement with previously published literature $[20,41]$. However, ATM precipitated at higher $\mathrm{pH}$, therefore it was important to maintain the $\mathrm{pH}$ of the HSA solution at 7 throughout the process. This could be explained by different HSA conformations at different $\mathrm{pH}$ values. The standard HSA conformation ' $\mathrm{N}$ ' is present at neutral $\mathrm{pH}$. A change to the ' $\mathrm{B}$ ' conformation occurs above $\mathrm{pH} 8$ which might have lower affinity to ATM [42]. Similar observations have been earlier reported [43].

High-pressure homogenization was used to prepare HSA nanoparticles [44]. Effect of homogenization pressure and number of homogenization cycles were seen to play an important role in determination of particle size. It was found that lower homogenization pressures were sufficient to get the desired particle size $(<100 \mathrm{~nm})$ and PI. Higher pressures led to increase in particle size (Supplementary Table 2). Though earlier reports have shown reduction in particle size with increase in homogenization pressure for HSA nanoparticles, a minimum particle size of $222.2 \mathrm{~nm}$ could only be achieved at high homogenization pressures [45].

\section{Freeze drying of nanoparticles}

AAN was freeze-dried to get a free flowing stable product. Freeze-drying of AAN without cryoprotectant and with mannitol and lactose as cryoprotectants resulted in increased particles size and PI (Figure 3) [46]. Trehalose at $3 \% \mathrm{w} / \mathrm{v}$ concentration was found useful as cryoprotectant for lyophilization of nanoparticles with neither a significant change in particle size (Figure $3 \mathrm{~A}$ ) nor in PI (Figure 3B). Trehalose is known to preserve the HSA nanoparticle during freeze-drying as it forms amorphous glass, which is reported to interact with amorphous protein in the freeze-dried cake [47].

\section{Fluorescence spectroscopy for determination of binding of ATM to HSA}

Fluorescence quenching of the single tryptophan residue in HSA was used to measure drug-binding affinity [48]. When HSA is excited at $280 \mathrm{~nm}$, fluorescence intensity around $350 \mathrm{~nm}$ reflects changes of the microenvironment of tryptophan residue. Addition of ATM showed dynamic quenching of the fluorescence of HSA (Supplementary Figure 4) and followed Stern Volume equation with Ksv of $1.755 \times 10^{4} 1 \mathrm{~mol}^{-1}$ [26]. A strong decrease in the fluorescence intensity in the spectra of AANs (650 a.u.) was observed in comparison with the BANs (900 a.u.) (Figure 4) suggesting interaction of ATM and HSA. The quenching process also signifies the tertiary structural changes in HSA demonstrating binding of drug with HSA in the nanoparticulate formulation [49].

\section{Characterization of the nanoparticles}

Mean particle size of AAN was found to be $66.1 \mathrm{~nm}$ (PI 0.090) by photon correlation spectroscopy and was in confirmation to that obtained from TEM and SEM (Figure 5) of the freeze-dried product (Figure 5D) with $\zeta$ potential of $-14.9 \mathrm{mV}$. TEM and SEM images both confirmed almost spherical morphology of AAN.

DSC thermogram of ATM and HSA exhibited endothermic peaks at 85.73 and $126.97^{\circ} \mathrm{C}$, respectively. AAN showed a shift in the peak to $160.45^{\circ} \mathrm{C}$ because of drug-albumin binding and formation of nanoparticles [20], although a small peak near the endothermic peak of the drug may be indicative of small amount of ATM present on the surface of HSA matrix (Supplementary Figure 1).

XRD pattern of ATM shows the presence of sharp peaks indicating the crystalline nature of the drug. The XRD of HSA exhibited a broad reflection with 
Table 3. Optimization of drug: human serum albumin ratio.

\begin{tabular}{|lllll|}
\hline Drug:HSA ratio & Particle size $(\mathbf{n m})$ & $\begin{array}{l}\text { Polydispersity } \\
\text { index }\end{array}$ & Observations & $\begin{array}{l}\text { Entrapment } \\
\text { efficiency (\%) }\end{array}$ \\
\hline $1: 20$ & 230.6 & 0.348 & Good drug dispersion & 68.45 \\
\hline $1: 10$ & 85.5 & 0.121 & Good drug dispersion & 77.56 \\
\hline $1: 5$ & - & & Precipitation of the drug & - \\
\hline
\end{tabular}

no distinct peaks revealing the amorphous structure of HSA. Absence of sharp peaks of ATM and broad humps in the AAN graph indicated that the drug was successfully incorporated into the HSA nanoparticles and was present primarily in an amorphous form within the nanoparticles (Supplementary Figure 2) [50]. However, some sporadic weak peaks in AAN might indicate that ATM might not be fully shielded by protein side chains and small amounts of drug may be present on the surface. This is in accordance with our DSC results and consistent with a previous study [51].

All the characteristic peaks of HSA observed in the FTIR were retained in the AAN indicating no chemical modification in the structure of the protein. The spectra of AAN revealed the characteristic peaks of ATM with almost negligible shifts from which it may be deduced that ATM is entrapped within the HSA matrix and that the chemical structure of ATM does not change after encapsulation (Supplementary Figure 3). Similar FTIR spectroscopy results have been demonstrated for encapsulation of bioactive molecules into albumin nanoparticles [40,52].

In vitro release curves (Figure 5) of ATM from AAN showed an initial burst effect followed by slow release. This could be because of the different ways in which the drug is loaded in the nanoparticles: adsorption and entrapment [53]. Lyophilization of nanoparticles did not have any effect on the drug release and was similar to that obtained from nanoparticles prior to lyophilization.

\section{In vitro erythrocyte toxicity study}

ATM being practically insoluble, required polysorbate 80 , a nonionic surfactant to be incorporated in the formulation to improve its solubility and drug entrapment. Surfactants may cause damage to cell membranes of erythrocytes due to increases in membrane permeability [54], and therefore an in vitro erythrocyte toxicity test was carried out to evaluate biocompatibility of the nanoparticles [55]. Triton-X 100 (positive control) showed $100 \%$ hemolysis of erythrocytes at the end of the incubation period. Though polysorbate 80 solution $(3 \% \mathrm{w} / \mathrm{v})$ exhibited hemolysis of $75.89 \%$ after $30 \mathrm{~min}$ incubation, AAN containing $3 \% \mathrm{w} / \mathrm{v}$ polysorbate 80 showed an erythrocyte hemolysis rate of only $9.92 \%$ (Figure 6), demonstrating its safety. An earlier report by Joshi et al. has shown very low erythrocyte hemolysis with ATM nanostructured lipid carrier (NLC) even at very high concentrations of surfactant [32]. This might be due to micelle formation of the surfactant at the nanoparticle and water interface, resulting in solubilization of the drug and stabilization of the entire system. Previous experiments have shown that a polysorbate 80 coating on the surface of HSA nanoparticles possess reduced toxicities and improved pharmacokinetic parameters of doxorubicin [56]. Clinically, upon administration, drugs injected intravenously are immediately diluted to a very large extent due to constant blood circulation and sink conditions provided by the body. To mimic in vivo conditions, diluted formulations were also tested for their hemolytic potential. AAN showed a hemolysis of $6.24 \%$ when diluted ten-times and $6.12 \%$ when diluted 100 -times at the end of $30 \mathrm{~min}$ (Figure 5).

\section{In vitro susceptibility assay}

Treatment of P. falciparum cultures with ATM and AAN demonstrated a dose-dependent antiparasitic effect while BAN, the HSA nanoparticle without any drug, failed to show any effect (Figure 7) [57]. AAN displayed optimal enhancement of antimalarial activity in a concentration range of 3.13 to $25 \mathrm{nM}$. At concentrations that were too low to effectively kill the parasite, insignificant differences were observed in the efficacy of drug solution and particles. Similarly, when drug concentrations were high enough to overcome rate-limiting diffusion and provide intracellular parasiticidal concentrations, the efficacy of particles was no different from that of the drug in solution. However, in a window of intermediate concentrations, the advantages of drug targeting using nanoparticles were unambiguously apparent. Maximum differences in inhibition of parasitemia was evident at 6.25 and $12.5 \mathrm{nM}$ drug concentrations of ATM demostrating significantly $(\mathrm{p}<0.001)$ higher antimalarial activity with AAN as compared to drug solution. $\mathrm{IC}_{50}$ values of AAN and ATM solution were 5.4 and $12.08 \mathrm{nM}$, respectively, evidencing improvement in efficacy at comparative $\mathrm{IC}_{50}$ dosages. 
(A)

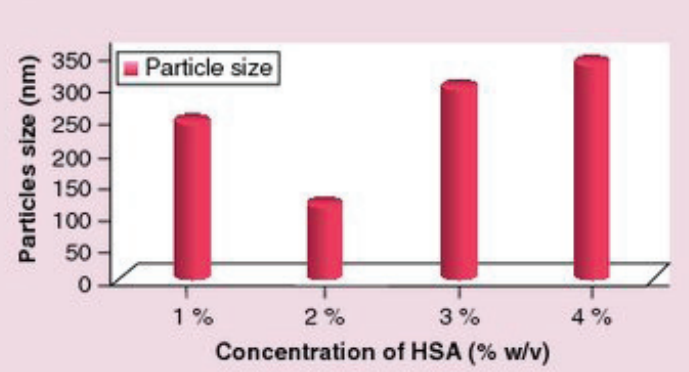

(c)
(B)

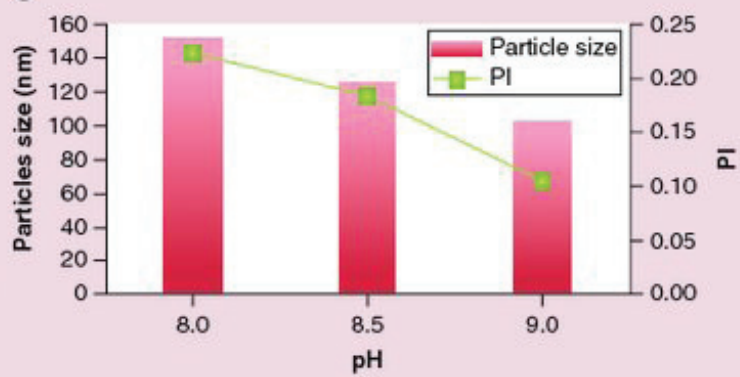

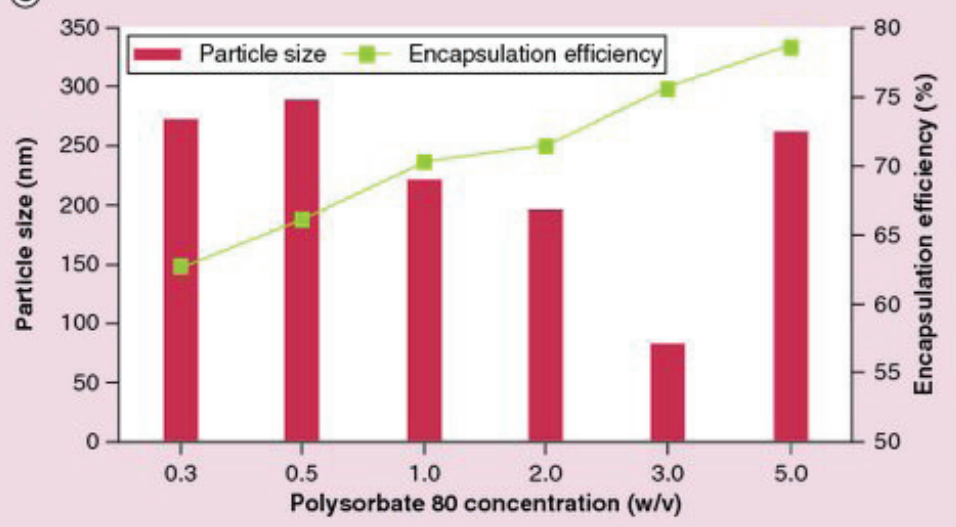

(E)
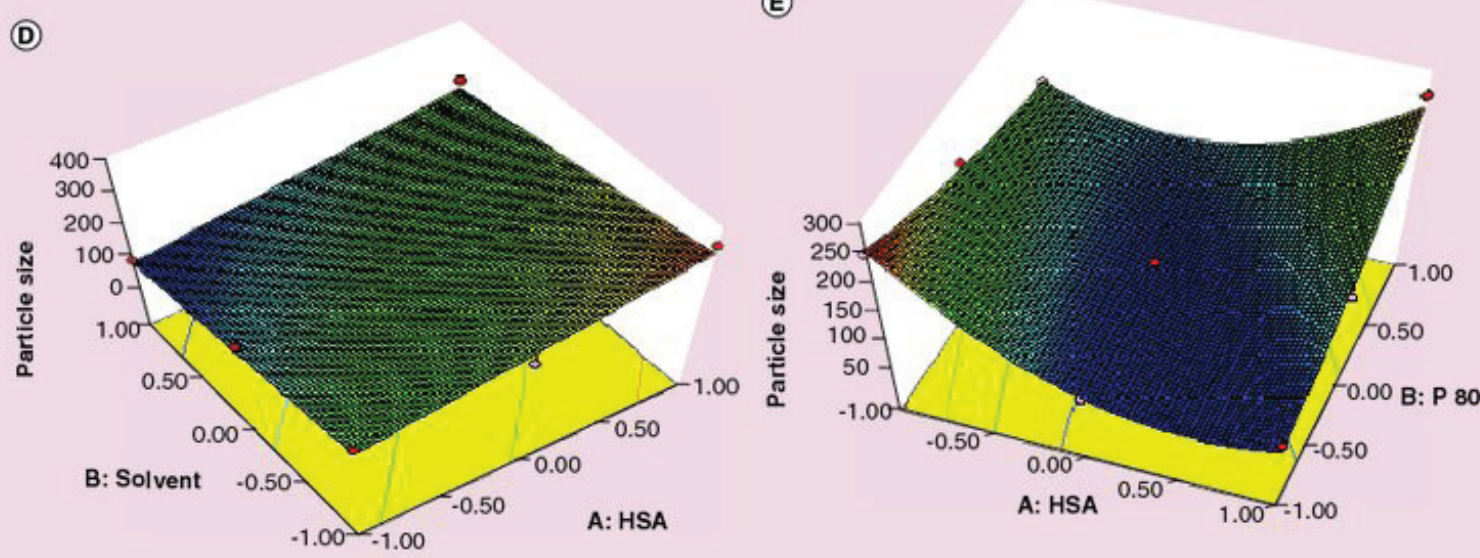

Figure 2. Optimization of human serum albumin nanoparticles. (A) Effect of human serum albumin (HSA) concentration on particle size of nanoparticles. (B) Effect of pH on particle size and polydispersity index of nanoparticles. (C) Effect of polysorbate 80 concentration on particle size and encapsulation efficiency of nanoparticles. (D) 3D Response surface plot showing the influence of HSA and solvent concentration on particle size of nanoparticles. (E) 3D Response surface plot showing the influence of HSA and surfactant concentration on particle size of nanoparticles.

HSA: Human serum albumin; P 80: Polysorbate 80.

\section{Determination of cellular uptake of}

nanoparticles by confocal microscopy imaging

Nanoparticles attached on the surface of erythrocytes possess extended circulation time in the body [58]. Nano-sized particles $(\leq 200 \mathrm{~nm})$ have been shown to enter the RBC; however, the translocation mechanism is not clear and remains controversial [59]. There- fore, study was carried out to analyze actual entry of the nanoparticles into the Plasmodium-infected erythrocytes.

DAPI staining helped in differentiating between parasitized and non-pRBC in the cocultures since DNA of the parasite is stained blue which is only present in the pRBC (Figure 8) [60]. Rhodamine- 


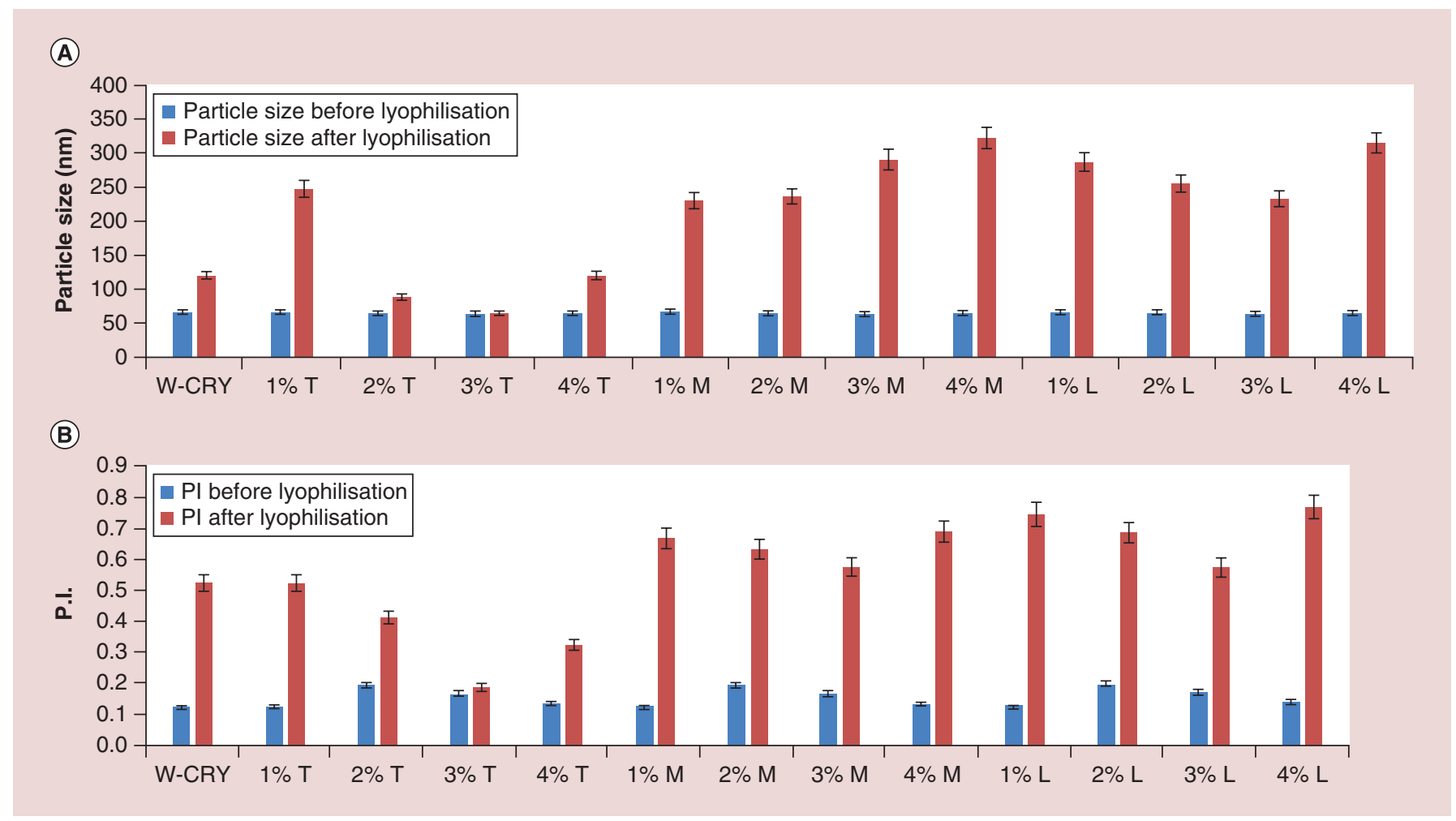

Figure 3. Effect of cryoprotectants on the (A) mean article size before and after lyophilization and (B) polydispersity index before and after lyophilization of artemether-loaded nanoparticle (mean $\pm S D, n=3$ ).

L: Lactose; M: Mannitol; PI: Polydispersity index; T: Trehelose; W-CRY: Without cryoprotectant.

labeled AAN incubated with Plasmodium-infected RBCs showed time-dependent uptake selectively by pRBCs as evident by colocated red fluorescence in DAPI stained cells (Figure 8). The uptake starting at as early as $5 \mathrm{~min}$ of incubation and the fluorescence was found to persist till the rupture of $\mathrm{pRBC}$. The uptake of nanoparticles was confirmed by Z-stacking of the confocal images that showed presence of fluo-

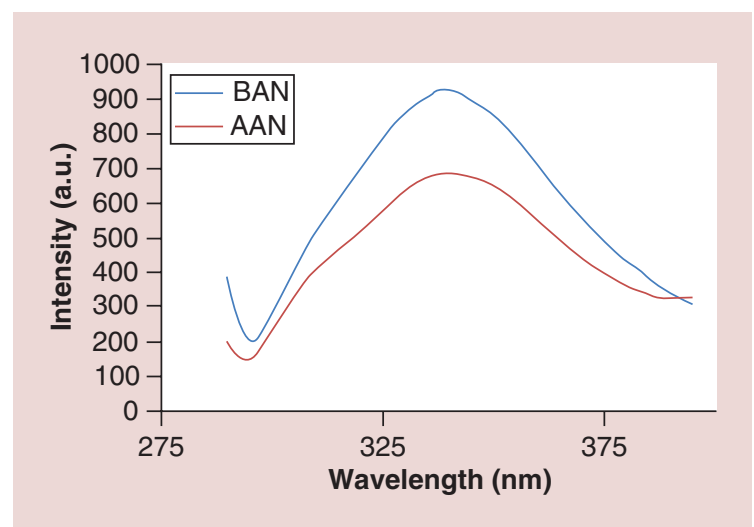

Figure 4. Fluorescence emission spectra of artemetherloaded nanoparticle and blank nanoparticle following excitation at $280 \mathrm{~nm}$.

AAN: Artemether-loaded HSA nanoparticle; BAN: Blank nanoparticle with HSA only. rescence due AAN in various sections of the pRBCs (Supplementary Figure 5). All the confocal images confirmed that nanoparticles do not penetrate the normal noninfected RBCs as was evident by absence of red fluorescence of rhodamine-labeled nanoparticles in these cells (Figure 8).

\section{In vivo antimalarial efficacy in $P$. berghei} infected mice

Nanostructured lipid carrier [32] and lipid nanoparticles [61] of ATM have shown improved antimalarial activity in $P$. berghei model. Peter's 4-day suppressive test was performed on AAN at two dose levels viz, 100 and $50 \%$ of therapeutic dose of the drug using $P$. berghei-infected Swiss albino mice. Due to absence of any intravenous injection for ATM, Larinate (artesunate injection), which is administered as intravenous injection was used as the reference.

The control group showed highest parasitemia at all-time intervals with maximum of $72.13 \%$ on day 10 with all mice found to be dead after 10th day. As compared with the control group, all the other groups showed reduction in the parasitemia during the course of study ( $\mathrm{p}<0.001)$, analyzed by one-way ANOVA followed by Tukey's multiple comparison test. Marketed formulation showed reduction in parasitemia $(23.46 \%$, 
(A)

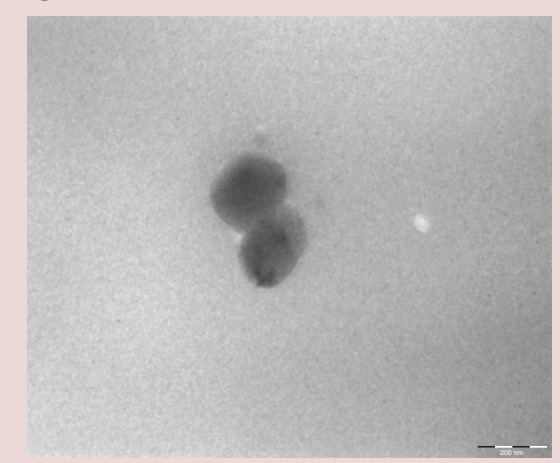

(E)

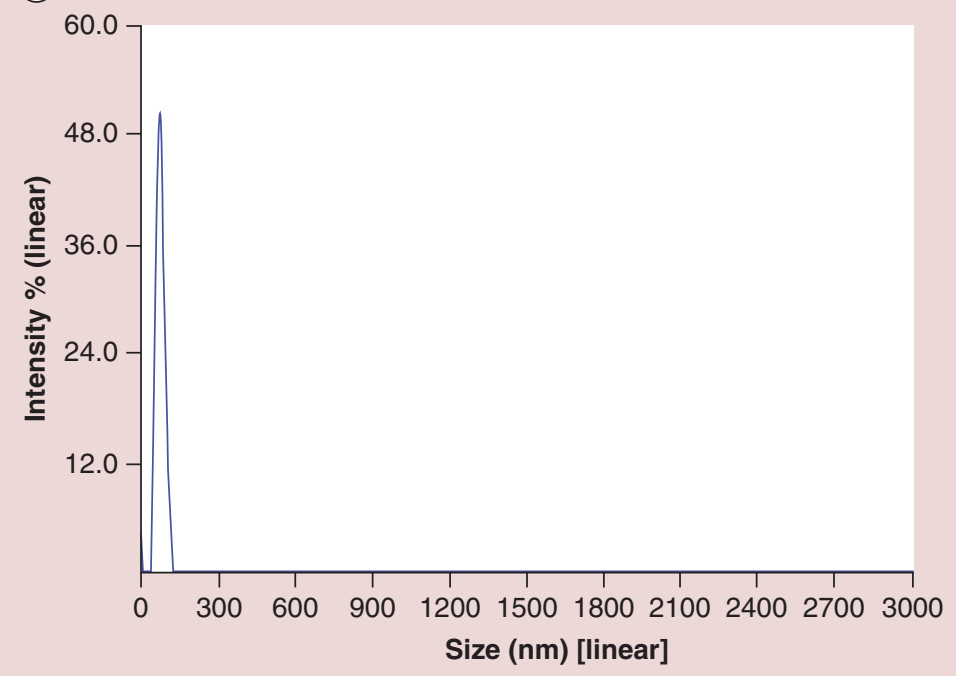

(B)

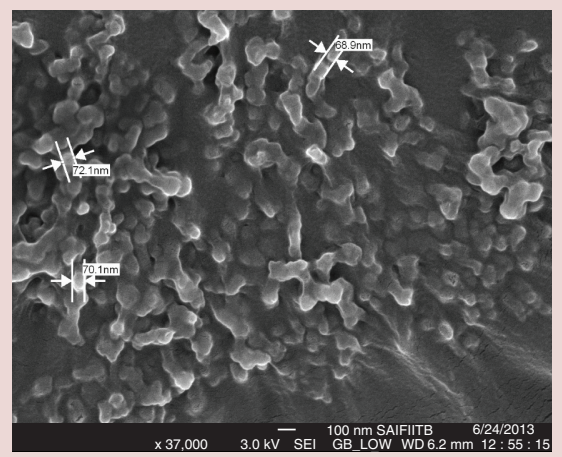

(C)

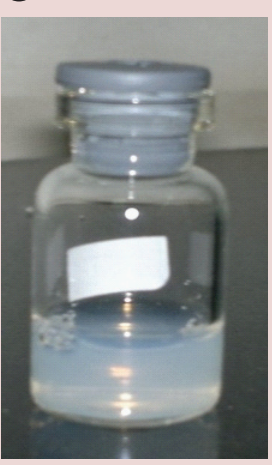

(D)

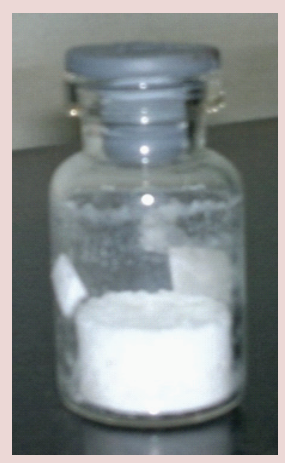

(F)

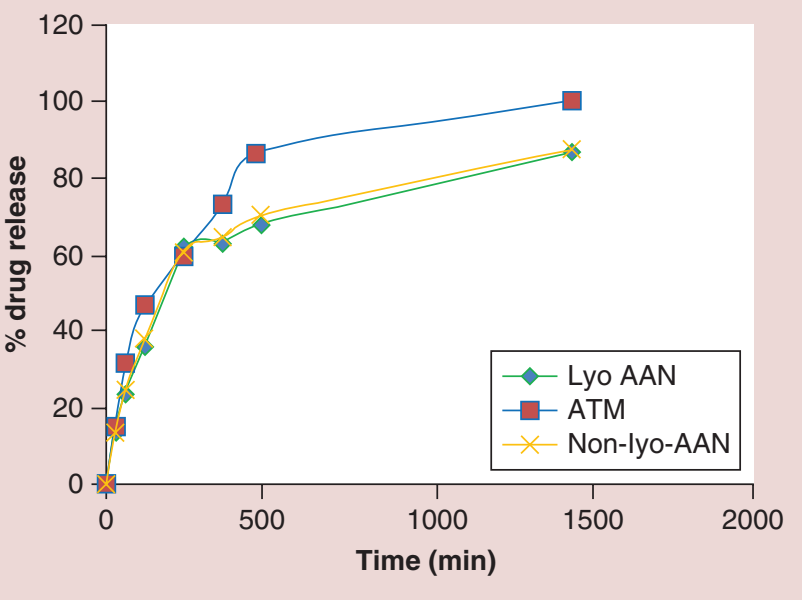

Figure 5. Characterization of artemether-loaded nanoparticle. (A) Transmission electron microscopic image of artemether-loaded nanoparticle (AAN). (B) Scanning electron microscopic image of AAN. (C) Photograph of AAN before lyophilization. (D) Photograph of AAN after lyophilization. (E) Representation of particle size distribution by intensity using photon correlation spectroscopy. (F) In vitro release profile of lyophilized and nonlyophilized AAN in phosphate buffered saline $(\mathrm{pH} 7.4)$ : ethanol $50: 50 \mathrm{v} / \mathrm{v}$ at $37^{\circ} \mathrm{C}(\mathrm{mean} \pm$ $S D, n=3)$.

ATM: Artemether; Lyo AAN: Lyophilized artemether-loaded HSA nanoparticle; Non-lyo-AAN: Artemether-loaded HSA nanoparticles prior to lyophilization.

day 21) as compared with control at $100 \%$ dose level; however, the reduction in parasitemia was much less (46.34\%, day 21) and the survival rate of animals was reduced to half (Table 4), when the dose was reduced to $50 \%$. Parasitemia with AAN was found to be $13.21 \%$ at $100 \%$ dose and $13.71 \%$ at $50 \%$ dose level at the end of 21st day of the experiment. AAN showed significantly less $(\mathrm{p}<0.001)$ parasitemia as compared with marketed intravenous injection of artesunate at both the dose levels (Figure 9). BANs showed reduction in the parasitemia (30.48\%) on 21st day as compared with the control. Previously Blank NLC (placebo vehicle) has also shown reduction in the parasitemia [32]. The reason for the activity is not known since none of the excipients is known to have any antimalarial activity. The surfactant used in the formula might by respon- sible for the parasitic cell hemolysis due to increased permeability of the parasitized cell, which might have resulted in the activity. Interestingly albumin has been reported to reduce mortality when given as adjunct therapy in children with severe malaria [62].

\section{Pharmacokinetics \& erythrocyte drug} concentration in $P$. berghei infected mice

AAN showed higher drug concentrations in the erythrocytes and deliver their payload to the target cell more efficiently than the molecular drug solution. AAN showed two-times higher peak drug concentration in the RBCs as compared with ATM solution (Figure 10A). This is consistent with the results obtained in in vitro $P$. falciparum studies, which showed increased intracellular uptake by $\mathrm{pRBCs}$ and higher inhibition of parasit- 


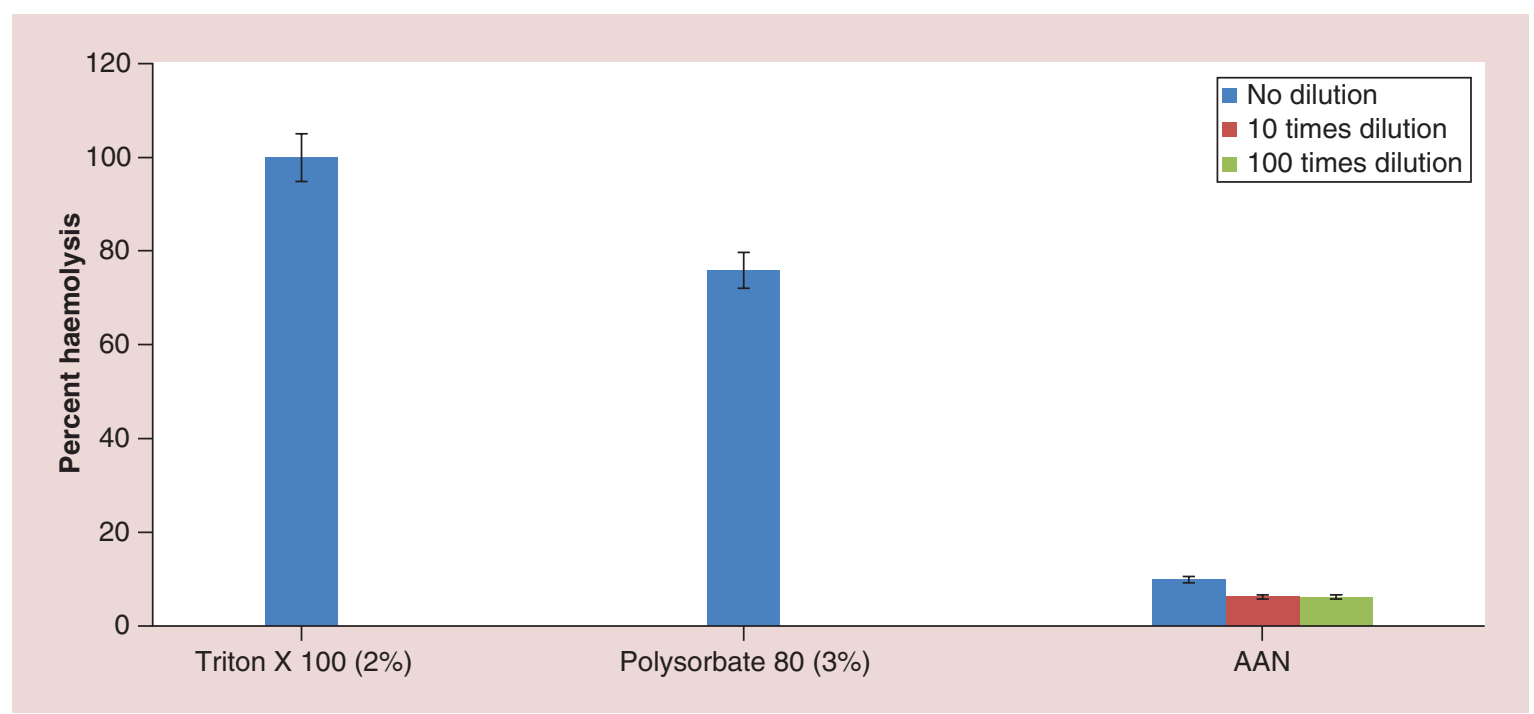

Figure 6. In vitro erythrocyte hemolysis after treatment with varying dilutions artemether-loaded nanoparticle in normal saline (mean $\pm S D, n=3)$. Triton-X $100(2 \% \mathrm{w} / \mathrm{v})$ in normal saline as positive control; Polysorbate 80 $(3 \% \mathrm{w} / \mathrm{v})$ in normal saline as reference.

AAN: Artemether-loaded HSA nanoparticle.

emia by AAN. This is also reflected by higher $\mathrm{AUC}_{0}{ }^{24}$ $(\mathrm{p}<0.05)$ in the RBCs after administration of AAN $(1017.5 \pm 107 \mu \mathrm{g} \mathrm{min} / \mathrm{ml})$ as compared with ATM solution $(554.5 \pm 68 \mu \mathrm{g} \mathrm{min} / \mathrm{ml}))$. After intravenous administration, AAN and ATM showed high initial plasma concentrations followed by constant decline with very low concentrations remaining at the end of $10 \mathrm{~h}$ and no drug in plasma at $24 \mathrm{~h}$. AAN revealed lower drug levels in plasma as compared with ATM probably because of partitioning more into RBCs (Figure 10B).

\section{Discussion}

Clinical management of severe and cerebral malaria

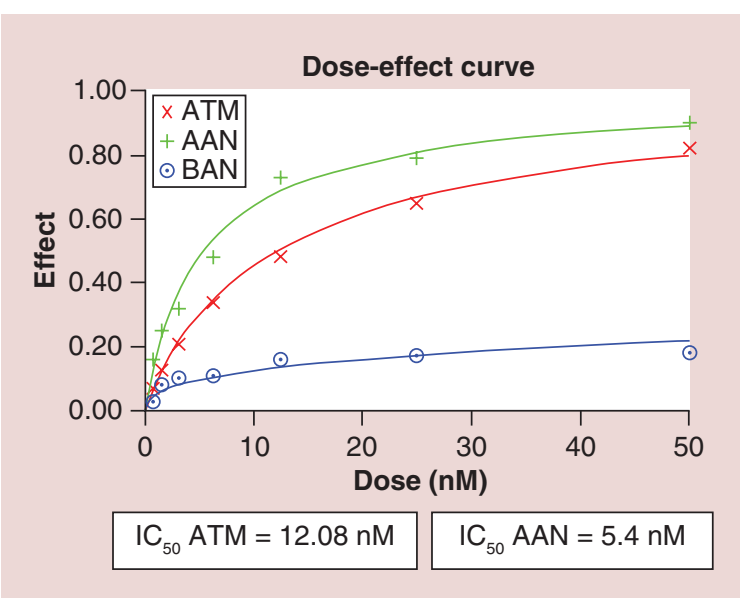

Figure 7. Dose response curves depicting mean parasitemia inhibition versus drug concentration $48 \mathrm{~h}$ after incubation in vitro (mean $\pm S D, n=3$ ).

AAN: Artemether-loaded HSA nanoparticle;

ATM: Artemether drug only; BAN: Blank nanoparticle with HSA only. remains a challenge with varying degrees of success even with parenteral administration of potent antimalarial agents. Drug delivery strategies that can target the antimalarial drugs selectively to the pRBCs can potentially reduce drug exposure to nontarget sites, delivering high drug concentrations to the infected cells and reducing the risk of drug resistance. In this study, we show HSA nanoparticles to be selectively taken up by $\mathrm{pRBCs}$ leading to higher local drug concentrations and improved antimalarial efficacy.

Robust HSA nanoparticles were developed using the $\mathrm{QbD}$ approach. An industrially feasible and scalable process of high-pressure homogenization was used to fabricate the nanoparticles [44]. ATM being water insoluble is presently available as an oily intramuscular injection. HSA showed strong binding to ATM (Figure 4F) and with resultant physical linkages facilitated nanoparticle formation. Addition of polysorbate $80(3 \% \mathrm{w} / \mathrm{v})$ stabilized the nanoparticles and prevented drug precipitation, leading to formulation of an aqueous injection of ATM with high drug loading [39]. Addition of polysorbate 80 had no discernible adverse effect on the cell membrane of erythrocytes, as demonstrated by low hemolytic activity $(<10 \%)$, which was further reduced on dilution (Figure 5). The nanoparticles can be considered safe for intravenous administration.

It is important to control the size of the prepared nanoparticles because smaller sized particles improve the bioavailability, with increased ability to enter cells. It has been reported that only NLCs $<400 \mathrm{~nm}$ diameter were taken up by pRBCs [63]. In another study, it was determined that fluorescent latex beads $>172 \mathrm{~nm}$ did not access intracellular parasites [7]. In our study, 


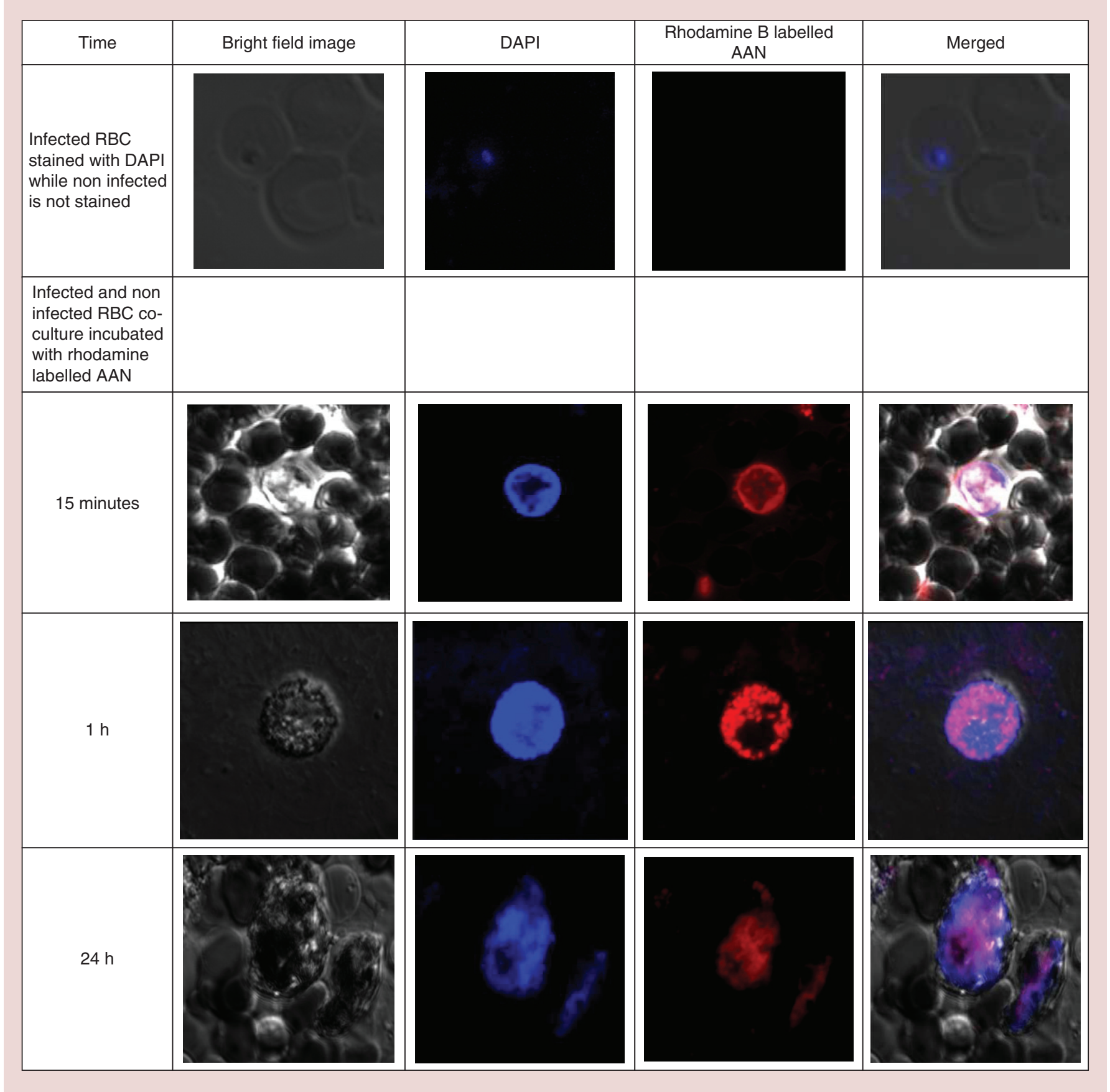

Figure 8. Confocal images showing selective targeting of rhodamine-labeled nanoparticles to parasitized red blood cells versus red blood cells. Rhodamine-labeled nanoparticles were added to living cocultures of $P$. falciparum infected red blood cells ( $p R B C s$ ) and noninfected RBCs, and incubated for the indicated times before sample preparation for microscopic analysis. Blue and red fluorescence represent DAPI (parasitized RBCs) and rhodamine B (localization of AAN), respectively. Figure panels $L$ to $R$ bright field image, DAPI stained Plasmodium nucleus showing parasitized RBC, Rhodamine-labelled artemether-loaded nanoparticles and superimposed images showing colocalization of Rhodamine-labelled AAN in parasitised RBCs.

AAN: Artemether-loaded HSA nanoparticle; DAPI: 4'6-diamino-2-phenylindole; RBC: Red blood cell.

we aimed to prepare nanoparticles having particle sizes less than $100 \mathrm{~nm}$. HSA concentration critically affected the particle size and drug entrapment. While higher HSA concentrations led to increase in particle size, lower concentrations decreased the drug entrapment. The average size of the particles also decreased with increase in the percentage of added ethanol [40,64]. In this context, the optimized nanoparticles with 
Table 4. Number of Plasmodium berghei-infected mice surviving in each group at the end of 30 th day $(n=5)$.

\begin{tabular}{|lll|}
\hline Treatment & Animals survived $(\mathbf{n})$ & Survival (\%) \\
\hline Untreated group & $0 / 5$ & 0 \\
\hline AAN $(0.624 \mathrm{mg} /$ day; $100 \%$ dose $)$ & $3 / 5$ & 60 \\
\hline AAN $(0.312 \mathrm{mg} /$ day; $50 \%$ dose) & $3 / 5$ & 60 \\
\hline $\begin{array}{l}\text { Larinate }(0.624 \mathrm{mg} / \text { day; } \\
100 \% \text { dose })\end{array}$ & $2 / 5$ & 40 \\
$\begin{array}{l}\text { Larinate }(0.312 \mathrm{mg} / \text { day; } \\
50 \% \text { dose })\end{array}$ & $1 / 5$ & 20 \\
BAN & $2 / 5$ & 40 \\
\hline AAN: Artemether-loaded HSA nanoparticle; BAN: Blank nanopartcle. & \\
\hline
\end{tabular}

Drug:HSA ratio of 1:10 at $40 \%$ ethanol concentration as desolvating agent produced the smallest nanoparticles with highest drug loading (77.6\%). Low homogenization pressure $200 \mathrm{bar}$ (ten cycles) followed by five cycles at 400 bar was sufficient to yield mean particle size of $66.1 \mathrm{~nm}$ and PI of 0.090. Higher homogenization pressures resulted in a larger particle size. This can be explained as follows; the additional energy input resulted in higher kinetic energy of the particles with subsequent coalescence. Once the maximum dispersitivity has been reached additional energy put into the system cannot be used for further dispersion of nanoparticles. The energy put in can only accelerate the velocity of the particles, giving them adequate kinetic energy to overcome the stabilization resulting in aggregation and increase in mean size [65].

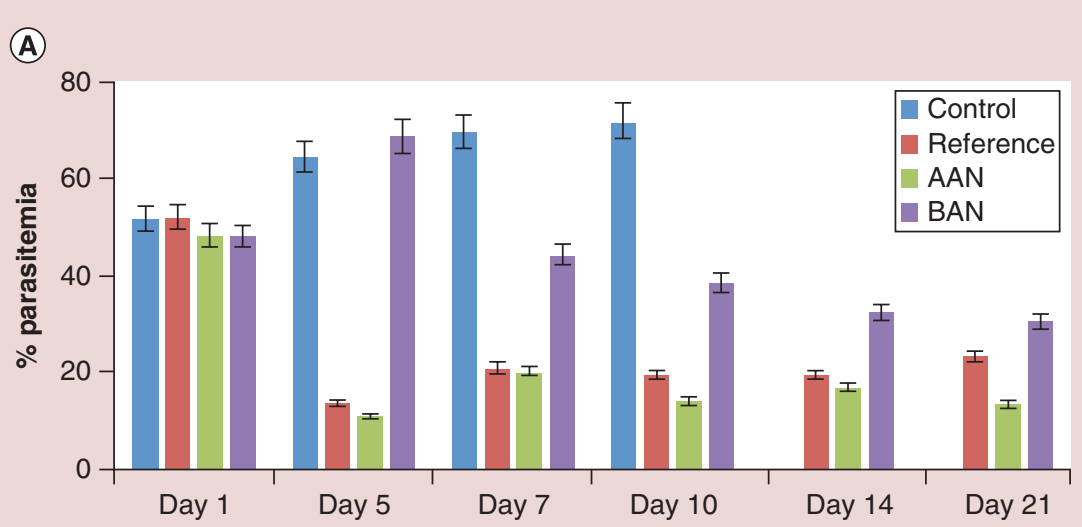

(B)

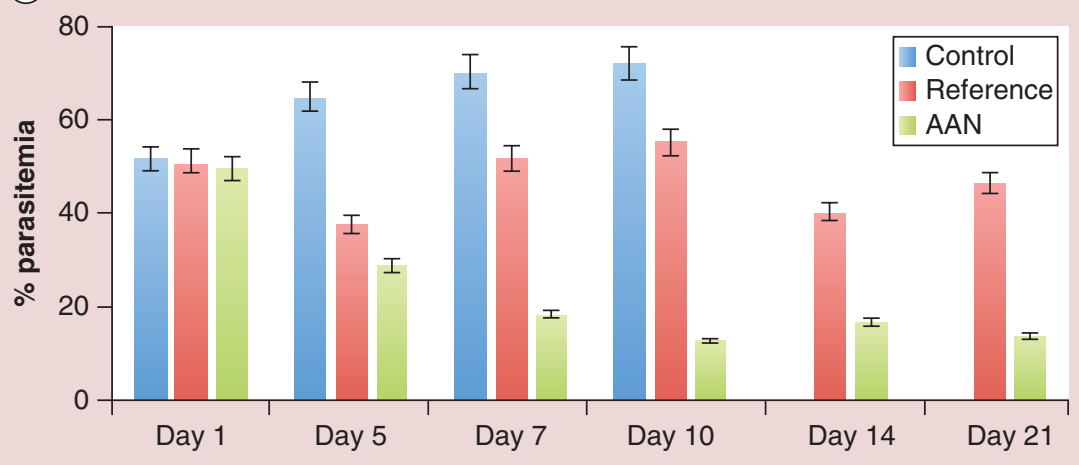

Figure 9. Mean percent parasitemia in suppressive test against Plasmodium berghei infected mice after treatment with artemether-loaded HSA nanoparticle, blank nanoparticle and Reference at (A) $100 \%$ (B) $50 \%$ dose levels. (values are expressed as mean \pm SD $n=5$ mice per group).

AAN: Artemether-loaded HSA nanoparticle; BAN: Blank nanoparticle with HSA only; Reference: Larinate artesunate marketed injection. 


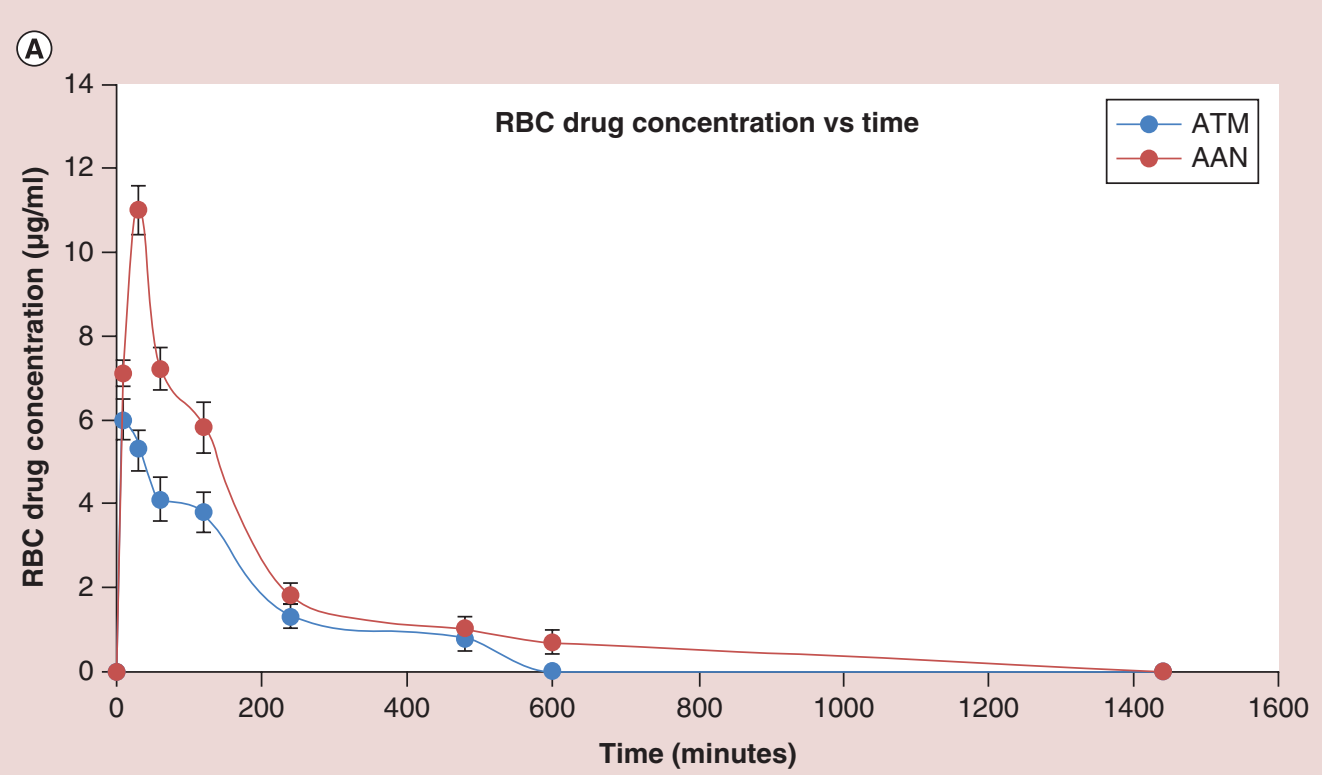

(B)

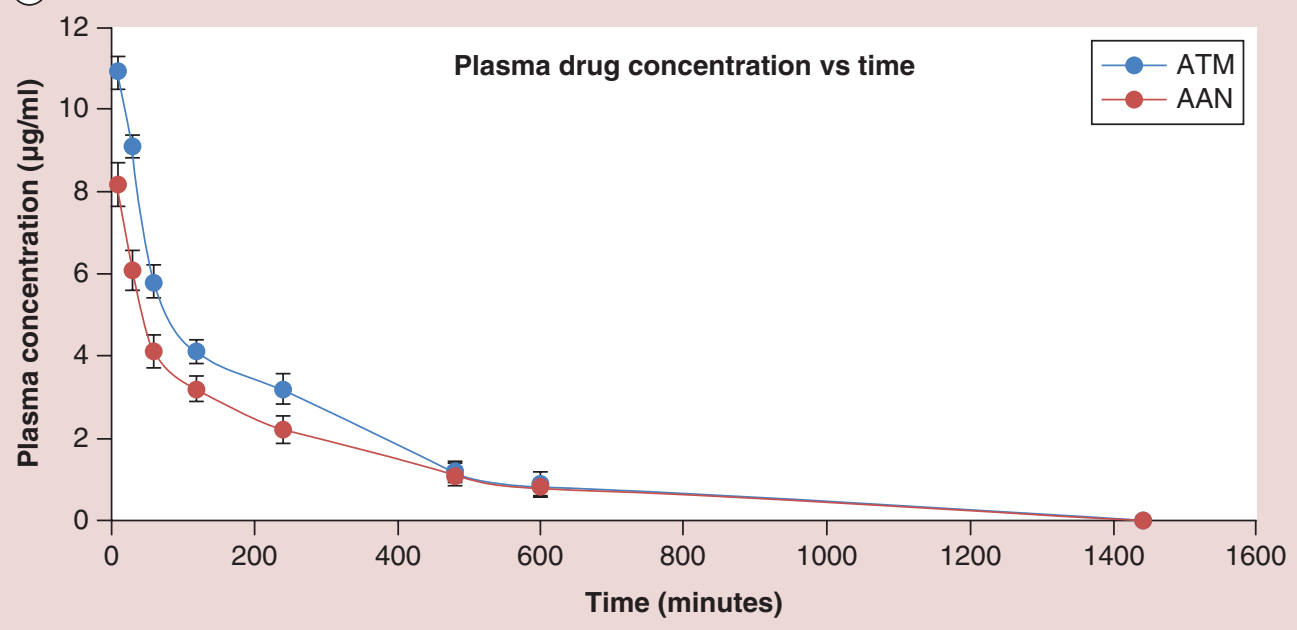

Figure 10. Drug concentration-time profiles in parasitized mice. (A) Red blood cell; (B) plasma ( $m e a n \pm S D, n=6$ ). AAN: Artemether-loaded HSA nanoparticles; ATM: Artemether drug only; RBC: Red blood cell.

Since ATM is known to be moisture sensitive, freezedrying was investigated to retain the stability of AAN. Freezing often induces many destabilizing stresses for nanoparticles. Formation of ice crystals that can mechanically destabilize nanoparticles and increased interaction due to enhanced nanoparticle concentration leading to aggregation or fusion are some of the phenomena observed during freeze drying [66]. Trehalose as an additive seems to provide sufficient protection from lyophilization of HSA protein, contributing to maintaining stability in the solid state [67]. Trehalose containing formulations were superior to mannitol and lactose, exhibiting minimal increases in nanoparticle size and PI following the freeze-drying procedure (Figure 3A \& B) [68].
The release of drug from AAN was characterized by a biphasic release pattern with an initial burst release $(\mathrm{t} 50 \%-4.6 \mathrm{~h})$ followed by sustained drug release ( $(90 \%-25.3 \mathrm{~h})$ (Figure 5E). The initial fast release is attributed to the weakly interacted drug associated with surface of the nanoparticles. Subsequently, the entrapped ATM released from the core of the nanoparticles in a sustained manner due to the diffusion of drug across the albumin matrix, would provide the possibility of continual drug effect. A similar phenomenon was observed previously in albumin nanoparticles [69-71]. However, the association of the drug with the carrier material in competition with other carriers such as plasma proteins, lipids and cell membranes plays an important role for the performance in vivo [72]. 
In vitro susceptibility assays in $P$. falciparum demonstrated significantly $(\mathrm{p}<0.001)$ higher antimalarial activity with AAN as compared to drug-only control as demonstrated by the lower $\mathrm{IC}_{50}$ values of AAN. This was confirmed by in vivo studies where AAN showed high antimalarial activity even at $50 \%$ dose in $P$. berghei infected mice. While the chemosuppression was reduced from 76.54 to $53.66 \%$, when free ARS dose was reduced to $50 \%$, chemosupression did not change significantly $(\mathrm{p}>0.05)$ showing equal activity (86.79 and $86.29 \%)$ at 100 and $50 \%$ doses respectively with AAN.

Confocal images clearly discriminated the selective uptake of Rhodamine-labeled AAN particles by pRBCs and not by normal RBCs. The amount of nanoparticles entering the $\mathrm{pRBC}$ was found to increase with time. It is a well-known fact that, mature erythrocytes do not display endocytosis and no pathways are evident for up taking macromolecules by these cells [73,74]. But contrary to this, uptake of ferritin claiming that Plasmodium-infected RBCs are capable of endocytosis have been reported [75]. Many changes occur in the host cell membrane during intracellular malarial parasite development with extensive degradation of spectrin, allowing endocytosis in pRBCs [76,77]. Parasites are capable of enhancing the permeability of cell membranes in the host through new permeability pathway [78], and tubovesicular membrane [79] allowing nutrients access to the parasite. This increased permeability differs from the native transport systems of the normal erythrocyte [80,81]. Membrane-impermeable macromolecules in the external medium have been shown to bypass the host cell cytosol to reach intracellular parasites [82,83]. Though internalization mechanisms remain to be elucidated, it is speculated that HSA nanoparticles would follow similar uptake pathways as HSA which has been reported to have direct access through the parasitophorous duct $[5,6,15]$ into the pRBCs as a nutritional requirement $[15,84,85]$. Pouvelle et al., have demonstrated the presence of tubular prominences, which develop in the cytoplasm of the $\mathrm{pRBC}$ that link up with the host cell membrane to form a transient or stable duct, providing a means of direct access between the parasite and the external milieu [86]. An alternative concept of a 'metabolic window' that forms during the ring stage of asexual parasite maturation, provides 'contact sites' between the host erythrocyte membrane and the PVM [87]. The altered membrane permeability coupled with capability for endocytosis could be key factors responsible for the entry of the nanoparticles selectively into the pRBCs $[2,5,75,80]$.

Previously, Hasan et al., reported on the entry of stearylamine liposomes into the $\mathrm{pRBC}$ when observed using fluorescent microscopy [88]. Choloroquine lipo- somes loaded with quantum dots, covalently functionalized with oriented, specific half-antibodies against P. falciparum have been found to dock to $\mathrm{pRBC}$ plasma membranes and release their cargo into the cell [89]. More recently polymers poly(amidoamines) AGMA1 and ISA23 have exhibited preferential binding to and internalization into $P$. falciparum and $P$. yoelii infected RBCs [90]. However, safety of these systems have not been definitively established. HSA being endogenous, stable, biocompatible and already commercially exploited for other applications has a distinct advantage over these nanosystems and could be a safer, new tool for targeting drugs to Plasmodium-infected cells. Improved activity and the associated reduction in parasitemia with AAN, could be attributed to parasite clearing as a result of selective cellular uptake of nanoparticles by $\mathrm{pRBC}$ as observed by confocal microscopy.

\section{Conclusion}

In conclusion, biodegradable ATM-loaded HSA-based nanoparticles were developed as an intravenous drug delivery system for the treatment of malaria. Robust formulation with reproducible particle size was successfully obtained employing the $\mathrm{QbD}$ approach. In vitro studies using $P$. falciparum showed a $50 \%$ reduction in $\mathrm{IC}_{50}$ values of AAN when compared with drug only controls. AAN showed marked less parasitemia as compared with marketed intravenous injection of artesunate and showed equivalent antimalarial activity at half the therapeutic dose. Uptake of nanoparticles by pRBCs was demonstrated by higher intracellular drug concentration in the erythrocytes as shown by the pharmacokinetic studies in the parasitized mice. Confocal imaging confirmed the selective entry of the nanoparticles into pRBCs, but not healthy uninfected RBCs. A follow-up quantitative fluorescence-assisted cell sorting analysis study is proposed as part of further work. Thus, HSA nanoparticles have shown significant potential to be used as a safe and effective drug delivery system for the treatment of malaria. As it permits intravenous administration and selective $\mathrm{pRBC}$ uptake, it can be a beneficial strategy in severe and cerebral malaria.

\section{Supplementary data}

To view the supplementary data that accompany this paper please visit the journal website at: www.futuremedicine.com/ doi/full/10.2217/nnm-2016-0235

\section{Financial \& competing interests disclosure}

Patent 2953/MUM/2013 has been filed and is pending. The authors have no other relevant affiliations or financial involvement with any organization or entity with a financial interest in or financial conflict with the subject matter or materials discussed in the manuscript apart from those disclosed. 
No writing assistance was utilized in the production of this manuscript.

\section{Ethical conduct of research}

The authors state that they have obtained appropriate institu- tional review board approval or have followed the principles outlined in the Declaration of Helsinki for all human or animal experimental investigations. In addition, for investigations involving human subjects, informed consent has been obtained from the participants involved.

\section{Executive summary}

- Biodegradable artemether-loaded human serum albumin (HSA) based nanoparticles (AANs) successfully developed as an intravenous drug delivery system for the treatment of malaria. Robust formulation optimized for critical product and process parameters with reproducible particle size $(<100 \mathrm{~nm})$ and high entrapment efficiency.

- Complete characterization carried out in terms morphology, particle size, polydispersity index, $\zeta$ potential, differential scanning calorimetry, x-ray diffraction, Fourier transform infrared spectroscopy and in vitro drug release.

- Fluorescence spectroscopy demonstrated binding of drug with HSA in the nanoparticulate formulation as seen by decrease in the fluorescence intensity in the spectra of AANs in comparison with the blank HSA nanoparticles.

- In vitro erythrocyte toxicity study established the safety for intravenous administration of AAN.

- Selective internalization of AAN into Plasmodium-infected red blood cells (parasitized RBCs) in preference to noninfected erythrocytes was observed using confocal imaging.

- In vitro susceptibility studies with Plasmodium falciparum showed $50 \%$ dose reduction for AAN as compared with drug-only controls to achieve $\mathrm{IC}_{50}$ levels of inhibition.

- The nanoparticles exhibited twofold higher peak drug concentrations in RBCs with antimalarial activity at $50 \%$ of therapeutic doses in Plasmodium bergei infected mice.

- HSA-based nanoparticles could offer a safe and effective approach for selective targeting of antimalarial drugs Plasmodium-infected cells. To best of our knowledge this is the first report of selective targeting of parasitozed RBCs with HSA-based nanoparticles.

\section{References}

1 WHO. World Malaria Report (2015). www.who.int/malaria/publications/world

2 Elford TB, Cowan GM, Ferguson DJP. Parasite-regulated membrane transport processes and metabolic control in malaria-infected erythrocytes. Biochem. J. 308, 361-374 (1995).

3 Kirk K, Tilley L, Ginsburg H. Transport and trafficking in the malaria-infected erythrocyte. Parasitol. Today 15 , 355-357 (1999).

4 Haldar K, Samuel BU, Mohandas N, Harrison T, Hiller NL. Transport mechanisms in Plasmodium-infected erythrocytes: lipid rafts and a tubovesicular network. Int. J. Parasitol. 12, 1393-1401 (2001).

5 Pouvelle P, Spiegel R, Hsiao L et al. Direct access to serum macromolecules by intraerythrocytic malaria parasites. Nature 353, 73-75 (1991).

6 Chairpan S, Przyborski JM. Protein transport across the parasitophorous vacuole of Plasmodium falciparum: into the great wide open. Traffic 9, 157-165 (2008).

7 Goodyer ID, Pouvelle B, Schneider TG, Trelka DP, Taraschi TF. Characterization of macromolecular transport pathways in malaria-infected erythrocytes. Mol. Biochem. Parasitol. 87, 13-28 (1997).

8 Aditya NP, Vathasal PG, Vierira V, Murthy RSR, Souto EB. Advances in nanomedicines for malaria treatment. $A d v$. Colloid Inter. Sci. 201-202, 1-17 (2013).

9 Singh KK. Nanomedicine in malaria. In: Patenting Nanomedicine: Legal Aspects. In Intellectual
Property and Grant Opportunities. Souto EB (Ed.). Springer-Verlag Berlin Heidelberg, Germany, 401-435 (2012).

10 Bannister LH, Hopkins JM, Fowler RE, Krishna S, Mitchell $\mathrm{GH}$. A brief illustrated guide to the ultrastructure of $P$. falciparum asexual blood stages. Parasitol. Today 16, 427-433 (2000).

11 Cortes J, Saura C. Nanoparticle albumin-bound (nab $\left.{ }^{\mathrm{TM}}\right)$ paclitaxel: improving efficacy and tolerability by targeted drug delivery in metastatic breast cancer. Eur. J. Cancer. Supplements 8, 1-10 (2010).

12 Elzoghby A, Samy W, Elgindy N. Albumin-based nanoparticles as potential controlled release drug delivery systems. J. Control. Rel. 157, 168-182 (2012).

13 Fasano M, Curry S, Terreno E. The extraordinary ligand binding properties of human serum albumin. IUBMB Life 57, 787-796 (2005).

14 Duranton C, Tanneur V, Lang C. A high specificity and affinity interaction with serum albumin stimulates an anion conductance in malaria-infected erythrocytes. Cell Physiol. Biochem. 2, 395-404 (2008).

15 Tahir AE, Malhotra P, Chauhan VS. Uptake of proteins and degradation of human serum albumin by Plasmodium falciparum-infected human erythrocytes. Malar. J. 2, 11 (2003).

16 Krishna S. Ulhemann A, Haynes RK. Artemisinins: mechanisms of action and potential for resistance. Drug Resist Updat. 7, 233-244 (2004). 
17 Artemether-monographs of antimalarial drugs. In: International Pharmacopoeia (3rd Edition). WHO, Geneva, Switzerland, 5, 187-190 (2003).

18 Yang YZ, Asawamahasakda W, Meshnick SR. Alkylation of human albumin by the antimalarial artemisinin. Biochem. Pharmacol. 46, 336-339 (1993).

19 Read M, Hyde JE. Simple in vitro culture of the malaria parasite Plasmodium falciparum (erythrocytic stages), suitable for large-scale preparations. Methods Mol. Biol. 21, 43-55 (1993).

20 Langer K, Balthasar S, Vogel V, Dinauer N, Briesen H, Schubert D. Optimization of the preparation process for Human serum albumin (HSA) nanoparticles. Int. J. Pharm. 257, 169-180 (2003)

21 Weber C, Coester C, Kreuter J, Langer K. Desolvation process and surface characterisation of protein nanoparticles. Int. J. Pharm. 194, 91-102 (2000).

22 Jun JY, Nguyen HH, Paik S, Chun HS, Kang B, Ko S. Preparation of size-controlled bovine serum albumin (BSA) nanoparticles by modified desolvation method. Food Chem. 127, 1892-1898 (2011).

23 Watcharin W, Schmithals C, Pleli T et al. Biodegradable human serum albumin nanoparticles as contrast agents for the detection of hepatocellular carcinoma by magnetic resonance imaging. Eur. J. Pharm. Biopharm. 87, 132-141 (2014).

24 Dubey RD, Alam N, Saneja A et al. Development and evaluation of folate functionalized albumin nanoparticles for targeted delivery of gemcitabine. Int. J. Pharm. 492, 80-91 (2015).

25 ICH Harmonised Tripartite Guideline, Pharmaceutical Development, Q8(R2) Current Step 4 version, dated August 2009 . www.ich.org

26 Ibrahim N, Ibrahim H, Kim S. Interactions between antimalarial indolone- $\mathrm{N}$-oxide derivatives and human serum albumin. Biomacromolecules 1, 3341-3351 (2010).

27 Bian Q, Liu J, Tian J, Hu Z. Binding of genistein to human serum albumin demonstrated using tryptophan fluorescence quenching. Int. J. Biol. Macromol. 34, 275-279 (2004).

28 Sharma P, Sharma J. In vitro hemolysis of human erythrocytes - by plant extracts with antiplasmodial activity. Ethnopharmacology 74, 239-243 (2001).

29 Wirjanata G, Sebayang BF, Chalfein F et al. Potent ex vivo activity of naphthoquine and methylene blue against drug-resistant clinical isolates of Plasmodium falciparum and Plasmodium vivax. Antimicrob. Agents Chemother. 59, 6117-6124 (2015).

30 Russell B, Chalfein F, Prasetyorini B. Determinants of in vitro drug susceptibility testing of Plasmodium vivax. Antimicrob. Agents Chemother. 52, 1040-1045 (2008).

31 Matthews H, Usman-Idris M, Khan F, Read M, Nirmalan N. Drug repositioning as a route to anti-malarial drug discovery: preliminary investigation of the in vitro antimalarial efficacy of emetine dihydrochloride hydrate. Malar. J. 12, 359 (2013).
32 Joshi M, Pathak S, Sharma S, Patravale V. Design and in vivo pharmacodynamic evaluation of nanostructured lipid carriers for parenteral delivery of artemether: nanoject. Int. J. Pharm. 364, 119-126 (2008).

33 Patil S, Joshi M, Pathak S, Sharma S, Patravale V. Intravenous b-artemether formulation (ARM NLC) as a superior alternative to commercial artesunate formulation. J. Antimicrob. Chemother. 67, 2713-2716 (2012).

34 Ibrahim N, Ibrahim H, Sabater AM et al. Artemisinin nanoformulation suitable for intravenous injection: preparation, characterization and antimalarial activities. Int. J. Pharm. 495, 671-679 (2015).

35 Matar KM, Awad AI, Elamin SB. Pharmacokinetics of artesunate alone and in combination with sulfadoxine/ pyrimethamine in healthy Sudanese volunteers. Am. J. Trop. Med. Hyg. 90 (6), 1087-1093 (2014).

36 Gad SC, Aubert N, Spainhour B et al. Nonclinical vehicle use in studies by multiple routes in multiple species. Int. J. Toxicol. 25(6), 499-521 (2006).

37 César IDC, Nogueira FHA, Pianetti GA. Simultaneous determination of artemether and lumefantrine in fixed dose combination tablets by HPLC with UV detection. J. Pharm. Biomed. Anal. 48, 951-954 (2008).

38 Shena Z, Ma G, Dobashib T, Maki Y, Su Z. Preparation and characterization of thermo-responsive albumin nanospheres. Int. J. Pharm. 346, 133-142 (2008).

39 Wang YJ, Wang C, Gong CY et al. Polysorbate 80 coated poly ( $\varepsilon$-caprolactone)-poly (ethylene glycol)-poly ( $\varepsilon$-caprolactone) micelles for paclitaxel delivery. Int. J. Pharm. 434, 1-8 (2012).

40 Ghosh P, Roy AS, Chaudhury S et al. Preparation of albumin based nanoparticles for delivery of fisetin and evaluation of its cytotoxic activity. Int. J. Biol. Macromol. 86, 408-417 (2016).

41 Langer K, Anhorna MG, Steinhausera I et al. Human serum albumin (HSA) nanoparticles: reproducibility of preparation process and kinetics of enzymatic degradation. Int. J. Pharm. 347, 109-117 (2008).

42 Carter DC, Ho JX, Structure of serum albumin. Adv. Protein Chem. 45, 153-203 (1994).

43 Dreisa S, Rothweilerb F, Michaelisb M, Cinatl J Jr., Kreutera J, Langer K. Preparation, characterisation and maintenance of drug efficacy of doxorubicin-loaded human serum albumin (HSA) nanoparticles. Int. J. Pharm. 341, 207-214 (2007).

44 Desai N, Tao C. US 6,749,868 B1 (2004).

45 Ibrahim N, Ibrahima H, Dormoic J et al. Albumin-bound nanoparticles of practically water-insoluble antimalarial lead greatly enhance its efficacy. Int. J. Pharm. 464, 214-224 (2014).

46 Anhorn M, Mahler H, Langer K. Freeze drying of human serum albumin (HSA) nanoparticles with different excipients. Int. J. Pharm. 363, 162-169 (2008).

47 Chang L, Shepherd D, Sun J. Mechanism of protein stabilization by sugars during freeze-drying and storage: native structure preservation, specific interaction, and/ or immobilization in a glassy matrix. J. Pharm. Sci. 94, 1427-1444 (2005). 
48 Trynda-Lemiesz L. Paclitaxel-HSA interaction. Binding sites on HSA molecule. Bioorg. Med. Chem. 12, 3269-3275 (2004).

49 Gong G, Pan Q, Wang K, Wu R, Sun Y, Lu Y. Curcuminincorporated albumin nanoparticles and its tumor image. Nanotechnology 26, 045603 (2015).

50 Wan X, Zheng X, Pang X, Zhang Z, Zhang O. Incorporation of lapatinib into human serum albumin nanoparticles with enhanced anti-tumor effects in HER2-positive breast cancer. Colloid Surface B. 136, 817-827 (2015).

51 Yang Z, Gong W, Wang Z et al. A novel drugpolyethylene glycol liquid compound method to prepare 10-hydroxycamptothecin loaded human serum albumin nanoparticle. Int. J. Pharm. 490, 412-428 (2015).

52 Bansal A, Kapoor DN, Kapil R, Chhabra N, Dhawan S. Design and development of paclitaxel-loaded bovin serum albumin nanoparticles for brain targeting. Acta Pharm. 61 (2), 141-156 (2011).

53 Kouchakzadeh Shojaosadati SA, Shokr F. Efficient loading and entrapment of tamoxifen in human serum albumin based nanoparticulate delivery system by a modified desolvation technique. Chem. Eng. Res. Des. 92, 1681-1692 (2014).

54 Gholamreza DN, Khezaeli P, Rahmani P. Study of the effects of polyethylene glycol sorbitan esters surfactants group on biological membrane. Int. J. Pharmacol. 4, 27-31 (2008).

55 Arora S, Rajwade J, Paknikar K. Nanotoxicology and in vitro studies: the need of the hour. Toxicol. Appl. Pharmacol. 258(2), 151-165 (2012).

Pereverzev E, Treschalin I, Bodyagin D et al. Influence of the formulation on the tolerance profile of nanoparticle-bound doxorubicin in healthy rats: focus on cardio- and testicular toxicity. Int. J. Pharm. 337, 346-356 (2007).

57 Tanariya P, Tippawangkoso P. In vitro sensitivity of Plasmodium falciparum and clinical response to lumefantrine (benflumetol) and artemether. J. Clin. Pharmacol. 49, 437-444 (2000).

58 Chambers E, Mitragotri S. Prolonged circulation of large polymeric nanoparticles by non-covalent adsorption on erythrocytes. J. Control. Rel. 10, 111-119 (2004).

59 Rothen-Rutishauser BSS, Haenni B, Kapp N, Gehr P. Interaction of fine particles and nanoparticles with red blood cells visualized with advanced microscopic techniques. Environ. Sci. Technol. 40, 4353-4359 (2006).

60 Baniecki ML, Wirth DF, Clardy J. High-throughput Plasmodium falciparum growth assay for malaria drug discovery. Antimicrob. Agents Chemother. 51, 716-723 (2007).

61 Aditya NP, Patankar S, Souto EB. Artemether-loaded lipid nanoparticles produced by modified thin-film hydration: pharmacokinetics, toxicological and in vivo antimalarial activity. Eur. J. Pharm. Sci. 40, 448-455 (2010).

62 John CC, Kutamba E, Mugarura K, Opoka RO. Adjunctive therapy for cerebral malaria and other severe forms of Plasmodium falciparum malaria. Expert Rev. Anti Infect. Ther. 8(9), 997-1008 (2010).
63 Jain S, Basu H, Prabhu P et al. Parasite impairment by targeting Plasmodium-infected RBCs using glyceryl-dilaurate nanostructured lipid carriers, Biomaterials 35, 6636-6645 (2014).

64 Teng Z, Luo Y, Wang Q. Nanoparticles synthesized from soy protein: preparation, characterization, and application for nutraceutical encapsulation. J. Agric. Food Chem. 60, 2712-2720 (2012).

65 Shegokar R, Singh KK, Müller RH. Production \& stability of stavudine solid lipid nanoparticles - from lab to industrial scale. Int. J. Pharm. 416, 2461-2470 (2011).

66 Abdelwahed H, Degobert G, Stainmesse S, Fessi H. Freezedrying of nanoparticles: formulation, process and storage considerations. Adv. Drug Deliv. Rev. 58, 1688-1713 (2006).

67 Dadparvara M, Wagnerb S, Wienb S et al. Freeze-drying of HI-6-loaded recombinant human serum albumin nanoparticles for improved storage stability. Eur. J. Pharm. Biopharm. 8, 510-517 (2014).

68 Han Y, Jin BS, Lee SB, Sohn Y, Joung JW, Lee JH. Effects of sugar additives on protein stability of recombinant human serum albumin during lyophilization and storage. Arch. Pharm. Res. 30 (9), 1124-1131 (2007).

69 Wilson B, Ambika TV, Patel RD, Jenita JL, Priyadarshini SR. Nanoparticles based on albumin: preparation, characterization and the use for 5 -flurouracil delivery. Int. J. Biol. Macromol. 51, 874-878 (2012).

70 Wilson B, Lavanya Y, Priyadarshini SR, Ramasamy M, Jenita JL. Albumin nanoparticles for the delivery of gabapentin: preparation, characterization and pharmacodynamic studies. Int. J. Pharm. 473, 73-79 (2014).

71 Wacker M. Nanocarriers for intravenous injection - the long hard road to the market. Int. J. Pharm. 457, 50-62 (2013).

72 Dubey RD, Alam N, Saneja A et al. Development and evaluation of folate functionalized albumin nanoparticles for targeted delivery of gemcitabine. Int. J. Pharm. 492, 80-91 (2015).

73 Haldar K, Uyetake L. The movement of fluorescent endocytic tracers in Plasmodium falciparum infected erythrocytes. Mol. Biochem. Parasitol. 50, 161-177 (1992).

74 Pouvelle B, Gormley JA, Taraschi TF. Characterization of trafficking pathways and membrane genesis in malaria-infected erythrocytes. Mol. Biochem. Parasitol. 6, 83-96 (1994).

75 Burns ER, Pollack S. P. falciparum infected erythrocytes are capable of endocytosis. In Vitro Cell Dev. Biol. 24, 481-486 (1988).

76 Ginsburg H, Stein WD. Biophysical analysis of novel transport pathways induced in red blood cell membranes. Mol. Biochem. Parasitol. 96, 1-10 (1987).

77 Yuthavong Y, Wilairat P, Panijpan B et al. Alteration in membrane proteins of mouse erythrocytes infected with different species and strains of malaria parasites. Comp. Biochem. Physiol. 63B, 83-85 (1979).

78 Ginsburg H, Stein WD. The new permeability pathways induced by the malaria parasite in the membrane of the infected erythrocyte: comparison of results using different experimental techniques. J. Membr. Biol. 197, 113-134 (2004) 
79 Lauer SA, Rathod PK, Ghori N, Haldar K. A membrane network for nutrient import in red cells infected with the malaria parasite. Science 276( 5315 ), 1122-1125 (1997).

80 Haldar K. Ducts, channels and transporters in Plasmodiuminfected erythrocytes. Parasitol. Today 10, 393-395 (1994).

81 Deponte M, Hoppe HC, Lee MC et al. Wherever I may roam: protein and membrane trafficking in $P$. falciparuminfected red blood cells. Mol. Biochem. Parasitol. 186(2), 95-116 (2012).

82 Saliba KJ, Kirk SK. Nutrient acquisition by intracellular apicomplexan parasites: staying in for dinner. Int. J. Parasitol. 31, 1321-1330 (2001).

83 Olliaro P, Castelli F. Plasmodium falciparum: an electronmicroscopy study of caveolae and trafficking between the parasite and the extracellular medium. Int. J. Parasitol. 27, 1007-1012 (1997).

84 Asahi H. Plasmodium falciparum: chemically defined medium for continuous intraerythrocytic growth using lipids and recombinant albumin Exp. Parasitol. 121, 22-28 (2009).

85 Asahia H, Kanazawab T, Hirayamac N, Kajiharad Y. Investigating serum factors promoting erythrocytic growth of Plasmodium falciparum. Exp. Parasitol. 109(1), 7-15 (2005)
86 Pouvelle B, Gysin J. Presence of the parasitophorous duct in Plasmodium falciparum and P. vivax parasitized Saimiri monkey red blood cells. Parasitol. Today 13, 357-361 (1997).

87 Bodammer JE, Bahr GF. The initiation of a "metabolic window" in the surface of host erythrocytes by Plasmodium berghei NYU-2. Lab. Invest. 28, 708-718 (1973).

88 Hasan GM, Garg N, Dogra E, Surolia R, Ghosh PC. Inhibition of the growth of Plasmodium falciparum in culture by stearylamine-phosphatidylcholine liposomes. J. Parasitol. Res. 2011, 120462 (2011).

89 Urbán P, Estelrich J, Cortés A, Fernàndez-Busquets X. A nanovector with complete discrimination for targeted delivery to Plasmodium falciparum-infected versus noninfected red blood cells in vitro. J. Control. Rel. 151, 202-211 (2011).

90 Movellan J, Urbán P, Moles E. Amphiphilic dendritic derivatives as nanocarriers for the targeted delivery of antimalarial drugs. Biomaterials 35, 7940-7950 (2014). 